\title{
THE OBSERVATIONS OF REDSHIFT EVOLUTION IN LARGE-SCALE ENVIRONMENTS (ORELSE) SURVEY. I. THE SURVEY DESIGN AND FIRST RESULTS ON CL 0023+0423 AT $z=0.84$ AND RX J1821.6+6827 AT $z=0.82$
}

\author{
L. M. Lubin ${ }^{1}$, R. R. Gal ${ }^{2}$, B. C. Lemaux ${ }^{1}$, D. D. Kocevski ${ }^{1}$, and G. K. Squires ${ }^{3}$ \\ ${ }^{1}$ Department of Physics, University of California-Davis, One Shields Avenue, Davis, CA 95616, USA; 1mlubin@ucdavis.edu \\ ${ }^{2}$ University of Hawaii, Institute for Astronomy, 2680 Woodlawn Drive, Honolulu, HI 96822, USA \\ ${ }^{3}$ California Institute of Technology, M/S 220-6, 1200 East California Boulevard, Pasadena, CA 91125, USA \\ Received 2008 August 26; accepted 2009 March 29; published 2009 April 29
}

\begin{abstract}
We present the Observations of Redshift Evolution in Large-Scale Environments (ORELSE) Survey, a systematic search for structure on scales greater than $10 h_{70}^{-1} \mathrm{Mpc}$ around 20 well-known clusters at redshifts of $0.6<z<1.3$. The goal of the survey is to examine a statistical sample of dynamically active clusters and large-scale structures in order to quantify galaxy properties over the full range of local and global environments. We describe the survey design, the cluster sample, and our extensive observational data covering at least $25^{\prime}$ around each target cluster. We use adaptively smoothed red galaxy density maps from our wide-field optical imaging to identify candidate groups/clusters and intermediate-density large-scale filaments/walls in each cluster field. Because photometric techniques (such as photometric redshifts, statistical overdensities, and richness estimates) can be highly uncertain, the crucial component of this survey is the unprecedented amount of spectroscopic coverage. We are using the wide-field, multiobject spectroscopic capabilities of the Deep Multiobject Imaging Spectrograph to obtain 100$200+$ confirmed cluster members in each field. Our survey has already discovered the $\mathrm{Cl} 1604$ supercluster at $z \approx 0.9$, a structure which contains at least eight groups and clusters and spans $13 \mathrm{Mpc} \times 100 \mathrm{Mpc}$. Here, we present the results on the large-scale environments of two additional clusters, $\mathrm{Cl} 0023+0423$ at $z=0.84$ and $\mathrm{RX}$ $\mathrm{J} 1821.6+6827$ at $z=0.82$, which highlight the diversity of global properties at these redshifts. The optically selected $\mathrm{Cl} 0023+0423$ is a four-way group-group merger with constituent groups having measured velocity dispersions between 206 and $479 \mathrm{~km} \mathrm{~s}^{-1}$. The galaxy population is dominated by blue, star-forming galaxies, with $80 \%$ of the confirmed members showing [O II] emission. The strength of the $\mathrm{H} \delta$ line in a composite spectrum of 138 members indicates a substantial contribution from recent starbursts to the overall galaxy population. In contrast, the X-rayselected RX J1821.6+6827 is a largely isolated, massive cluster with a measured velocity dispersion of $926 \pm 77$ $\mathrm{km} \mathrm{s}^{-1}$. The cluster exhibits a well-defined red sequence with a large quiescent galaxy population. The results from these two targets, along with preliminary findings on other ORELSE clusters, suggest that optical selection may be more effective than X-ray surveys at detecting less-evolved, dynamically active systems at these redshifts.
\end{abstract}

Key words: catalogs - galaxies: clusters: general - large-scale structure of universe - surveys

Online-only material: color figures

\section{INTRODUCTION}

Clusters of galaxies are accurate tracers of the large-scale structure in the local universe. Redshift surveys at $z<0.1$ (e.g., Geller \& Huchra 1989; Einasto et al. 1997; Colless et al. 2001) and numerical simulations (e.g., Colberg et al. 2000; Evrard et al. 2002; Dolag et al. 2006) reveal the filamentary structure of the universe, stretching between clusters and superclusters of galaxies. Clusters form at the nodes of these filaments, growing through the continuous accretion of individual galaxies and groups from the surrounding field (e.g., Frenk et al. 1996; Eke et al. 1998). The precursors of the filaments should be present around distant clusters, containing many of the galaxies which will eventually infall into the virialized core and form the cluster population observed today. Since galaxy environment should change dramatically during the course of vigorous cluster assembly, the large-scale structure present around high-redshift clusters offers us the unique opportunity to probe, over the full range of local environments, the physical effects on galaxies as they assemble into denser regions. As a result, we are undertaking the Observations of Redshift Evolution in LargeScale Environments (ORELSE) Survey, a systematic search for structure on scales greater than $10 h_{70}^{-1} \mathrm{Mpc}$ around 20 known clusters at $z>0.6$. The survey covers $5 \mathrm{deg}^{2}$, all targeted at overdense cluster regions, making it complementary and comparable to field surveys such as DEEP2 (Davis et al. 2003) and COSMOS (Scoville et al. 2007; Koekemoer et al. 2007).

While superclusters have been studied at low redshift (Davis et al. 1980; Postman et al. 1988; Quintana et al. 1995; Small et al. 1998; Rines et al. 2002; Fadda et al. 2008; Porter et al. 2008) and at $z \approx 0.2-0.6$ (Kaiser et al. 1999; Gray et al. 2002; Kodama et al. 2001, 2003, 2005; Ebeling et al. 2004; Tanaka et al. 2007a; Kartaltepe et al. 2008), large-scale structures at high redshift $(z \geqslant 0.6)$ are just now being explored (Kodama et al. 2005; Nakata et al. 2005; Tanaka et al. 2006, 2007b; Swinbank et al. 2007; Gal et al. 2008; Fassbender et al. 2008; Gilbank et al. 2008; Patel et al. 2009). Previous studies at these redshifts have largely focused on the central regions (less than $2 h_{70}^{-1} \mathrm{Mpc}$ ) of massive clusters. These studies have revealed strong evolution in the cluster galaxy population which includes (1) an increase in the fraction of blue, star-forming, late-type galaxies with redshift, implying that early types are forming out of the excess of late types over the last $\sim 7$ Gyr (e.g., Butcher \& Oemler 1984; Ellingson et al. 2001; Dressler et al. 1997; van Dokkum et al. 2000, 2001; Lubin et al. 2002); (2) a larger 
fraction of poststarburst $(\mathrm{K}+\mathrm{A})$ galaxies in the cluster versus field environment, indicating strong star formation activity in the recent past (e.g., Dressler et al. 1999, 2004; Balogh et al. 1999; Tran et al. 2003); (3) a deficit of faint, passive, red galaxies, suggesting that a large fraction of these galaxies in present-day clusters has moved onto the red sequence relatively recently as a result of a truncation of star formation (e.g., Smail et al. 1998; van Dokkum \& Franx 2001; Kodama et al. 2004; De Lucia et al. 2004, 2007; Tanaka et al. 2005, 2007b; Koyama et al. 2007); and (4) an increase in the overdensities of Xray and radio sources with cluster redshift, indicating enhanced starburst/nuclear activity in the past (e.g., Best 2003; Cappelluti et al. 2005; Eastman et al. 2007; Kocevski et al. 2009a).

All of these findings imply significantly increased starforming, starburst, and nuclear emission in the past and raise the question-What physical mechanisms associated with the cluster environment are responsible for the suppression of star formation and nuclear activity and the transformation of gaseous, disk galaxies into passive spheroids? Several mechanisms have been suggested, including galaxy harassment (Moore et al. 1998), ram pressure stripping (Gunn \& Gott 1972), starvation (Larson et al. 1980), and merging (Mihos 1995, 1999). Most of these processes are associated not with the densest cluster regions, but rather with the infall regions and lower density environments far from the cluster cores.

Studies at low to moderate redshift suggest that the noncluster processes play a pivotal role in driving galaxy evolution well before the galaxies reach the cluster cores. Data from the Sloan Digital Sky Survey (SDSS) and the Two-Degree Field (2dF) Survey indicate a sharp transition between galaxies with fieldlike star formation rates (SFRs) and galaxies with low SFRs comparable to that of galaxies within the cluster cores (Lewis et al. 2002; Gómez et al. 2003; Goto et al. 2003a, 2003b; Balogh et al. 2004). The density at which this transition occurs $\left(\log \Sigma \sim 1 \mathrm{Mpc}^{-2}\right.$ ) corresponds to the cluster virial radius, well outside the core region on which most studies have been focused. The fact that low SFRs and passive (gas-less) spiral galaxies are observed well beyond the virial radius rule out severe processes, such as ram pressure stripping or galaxy-galaxy merging, as being completely responsible for the variations in galaxy properties with environment (Lewis et al. 2002; Goto et al. 2003a). Similarly, studies on large scales (clustocentric distances of $>4 h_{70}^{-1} \mathrm{Mpc}$ ) at $z \sim 0.4$ indicate that galaxy colors change sharply at relatively low densities and that the morphological mix, at a given density, is independent of cluster radius, implying that galaxy transformation occurs outside the cluster core and is physically associated with the infalling filaments and the chains of smaller galaxy groups within them (Kodama et al. 2001; Treu et al. 2003). Consequently, these groups serve as a preprocessing phase in the evolution of cluster galaxies (Kodama et al. 2001, 2003, 2004; Gray et al. 2002; Treu et al. 2003; Bower \& Balogh 2004; Mihos 2004).

These trends continue to redshifts approaching $z \sim 1$ where there are indications of "downsizing" with galaxy evolution accelerated in high-density and high-mass (cluster) regions, compared to lower density and lower mass (group) regions. Studies of large-scale structures at $z \sim 0.5-0.8$ suggest that the galaxy population in clusters is more evolved, with the faint end of the red sequence being more developed and the red sequence galaxies showing no signs of current or recent star formation. In contrast, groups and lower density environments have a stronger deficit of the faint red sequence galaxies and clear signs of star formation activity (weak [O II] emission and/ or strong $\mathrm{H} \delta$ absorption) in the existing red galaxy population (De Lucia et al. 2004, 2007; Kodama et al. 2004; Tanaka et al. 2004, 2005; Koyama et al. 2007). As such, these results confirm that, at these epochs, much of the activity is taking place in lower density, lower mass environments.

This environment-activity level connection also extends to the extremes of active galactic nuclei (AGNs) and starburst galaxies. From studies covering a wide redshift range $(0.2<$ $z<1.2$ ), excesses of $24 \mu \mathrm{m}$ and X-ray sources are observed preferentially in intermediate-density, not high-density, regimes, consisting of groups, cluster infall regions, and filaments (Cappelluti et al. 2005; Gilmour et al. 2007; Marcillac et al. 2007, 2008; Kocevski et al. 2009b). These results, as well as those described above, imply that the environmental processes which induce nuclear activity, truncate star formation, and change galaxy properties are not necessarily driven by cluster-specific mechanisms, such as ram pressure stripping by the hot intracluster medium or harassment (i.e., truncation of the galaxy halo) by the cluster tidal field. Thus, it is essential to look well beyond the regions of traditional study (the cluster cores) to find answers to our questions concerning galaxy evolution. The ORELSE Survey is designed specifically to target these largely unexplored regions and answer these questions by correlating multiwavelength (radio, optical, infrared, and X-ray) photometric data with galaxy kinematics and spectral features in a statistical sample of large-scale structures at redshifts approaching unity.

The first large-scale structure detected in the ORELSE Survey was the $\mathrm{Cl} 1604$ supercluster at $z \approx 0.9$ which includes two massive clusters $\mathrm{Cl} 1604+4304$ at $z=0.90$ and $\mathrm{Cl}$ $1604+4321$ at $z=0.92$ originally detected by Gunn et al. (1986) and further studied by Oke et al. (1998). Wide-field imaging and spectroscopy as part of the ORELSE Survey has revealed a more complex, massive structure, containing at least eight groups and clusters and spanning $13 \mathrm{Mpc} \times$ 100 Mpc (Lubin et al. 2000; Gal \& Lubin 2004; Gal et al. 2005, 2008). Our extensive spectroscopic data on the $\mathrm{Cl} 1604$ supercluster (over 400 confirmed members) demonstrate that comprehensive redshift surveys, such as ORELSE, are essential for understanding galaxy and cluster evolution. Specifically, (1) the entire structure size in redshift space is equivalent to typical photometric redshift errors (Margoniner \& Wittman 2008); (2) superpositions of groups/clusters mean that mass measures based on weak lensing signal, richness, or X-ray luminosity are highly uncertain; (3) cluster velocity dispersions based even on traditionally large numbers of galaxies (i.e., 20-40) can be substantially overestimated due to outliers (Gal et al. 2008); and (4) the expected overdensities of radio and X-ray sources are small, making the identification and study of individual active galaxies impossible (Kocevski et al. 2009a).

In this paper, we build on our studies of the $\mathrm{Cl} 1604$ supercluster. We present the experimental design of the full survey (Section 2) and the photometric and spectroscopic results on two additional target clusters, $\mathrm{Cl} 0023+0423$ (hereafter $\mathrm{Cl}$ 0023) at $z=0.84$ and RX J1821.6+6827 (hereafter RX J1821) at $z=0.82$, which highlight the significant diversity of structure properties at these redshifts (Sections 4 and 5). Throughout the paper, we use a cosmology with $H_{0}=70 h_{70}^{-1} \mathrm{~km} \mathrm{~s}^{-1} \mathrm{Mpc}^{-1}$, $\Omega_{m}=0.3$, and $\Omega_{\Lambda}=0.7$.

\section{THE SURVEY DESIGN}

The ORELSE Survey is a systematic search for structures on scales greater than $10 h_{70}^{-1} \mathrm{Mpc}$ around 20 well-studied 
Table 1

The Cluster Sample

\begin{tabular}{|c|c|c|c|c|c|c|c|c|c|}
\hline Cluster & R.A. (J2000) & Decl. (J2000) & $\bar{z}$ & Selection & $\begin{array}{c}\text { Chandra/XMM-Newton } \\
\text { Observations? }\end{array}$ & $\begin{array}{c}\text { Optical } \\
\text { Complete? }\end{array}$ & $\begin{array}{c}\text { Near-infrared } \\
\text { Complete? }\end{array}$ & Notes & References \\
\hline $\mathrm{Cl} 0023+0423$ & 002352.2 & +042307 & 0.845 & Optical & Yes & Yes & Partial & Complex merger & 1 \\
\hline RCS J0224-0002 & 022430.0 & -000200 & 0.772 & Optical & Yes & & & Strong lens & 2 \\
\hline $\mathrm{Cl} \mathrm{J0849+4452}$ & 084856.3 & +445216 & 1.261 & $\mathrm{X}$-ray & Yes & Yes $^{\mathrm{b}}$ & & Lynx double cluster & 4 \\
\hline $\mathrm{Cl} \mathrm{J} 0910+5422$ & 091044.9 & +542209 & 1.110 & X-ray & Yes & Yes & Yes & Elongated structure & 5 \\
\hline Cl 0934+4804 & 094341.0 & +480446 & 0.699 & Optical & Yes & Yes & Yes & & 6 \\
\hline RX J1221.4+4918 & 122124.5 & +491813 & 0.700 & X-ray & Yes & Yes & Yes & Double cluster & 9 \\
\hline $\mathrm{Cl} 1324+3011$ & 132450.4 & +301126 & 0.756 & Optical & Yes & Yes & Yes & Supercluster member & 10 \\
\hline $\mathrm{Cl} 1324+3059$ & 132453.5 & +305900 & 0.755 & Optical & Yes & Yes & Yes & Supercluster member & 11 \\
\hline $\mathrm{Cl} 1325+3009$ & 132518.7 & +300957 & 1.067 & Optical & & Yes $^{b}$ & Yes & & 12 \\
\hline $\mathrm{Cl} \mathrm{J} 1350+6007$ & 135048.5 & +600707 & 0.804 & X-ray & Yes & Yes & Yes & Disturbed, elliptical structure & 13 \\
\hline Cl J1429.0+4241 & 142906.4 & +424110 & 0.920 & $\mathrm{X}$-ray & Yes & Yes & Yes & Elongated structure & 14 \\
\hline RX J1821.6+6827 & 182132.9 & +682755 & 0.818 & X-ray & Yes & Yes & Yes & Elongated structure & 20 \\
\hline
\end{tabular}

\section{Notes.}

${ }^{a}$ Archival data from the CFHTLS (see http://www. cfht.hawaii.edu/Science/CFHTLS/).

${ }^{b}$ Archival data from Subaru (see http://smoka.nao.ac.jp/).

References. 1. Gunn et al. (1986); Oke et al. (1998); 2. Gladders \& Yee (2000); Gladders et al. (2002); 3. Valtchanov et al. (2004); Andreon et al. (2005); Pierre et al. (2006); 4. Rosati et al. (1998, 1999); Nakata et al. (2005); Mei et al. (2006a); 5. Stanford et al. (2002); Mei et al. (2006b); 6. Gunn et al. (1986); Oke et al. (1998); 7. Hashimoto et al. (2005); 8. Blanton et al. (2003a); 9. Vikhlinin et al. (1998); Mullis et al. (2003); Jeltema et al. (2005); 10. Gunn et al. (1986); Oke et al. (1998); 11. Gunn et al. (1986); Oke et al. (1998); 12. Postman et al. (1996); Oke et al. (1998); 13. Rosati et al. (1998); Holden et al. (2002); Jeltema et al. (2005); 14. Maughan et al. (2006); 15. Gunn et al. (1986); Oke et al. (1998); 16. Gunn et al. (1986); Oke et al. (1998); 17. Gioia et al. (1999); Jeltema et al. (2005); 18. Gioia et al. (2003); Henry et al. (2006); 19. Gioia et al. (2003); Henry et al. (2006); 20. Gioia et al. (2003, 2004); Henry et al. (2006).

clusters at redshifts of $0.6<z<1.3$ (see Table 1). The goal of the ORELSE Survey is to study the large-scale structure around a statistical sample of high-redshift clusters. Numerical simulations indicate that $50 \%$ of all $M>3 \times 10^{14} h_{70}^{-1} M_{\odot}$ clusters at $z \sim 0.8$ have two or more companion clusters of equal or greater mass within 100 co-moving Mpc (i.e., a configuration similar to the $\mathrm{Cl} 1604$ supercluster), and 70\% have had its most recent large merger $\left(M_{\text {final }} / M_{\text {initial }} \geqslant 1.2\right)$ within the last $\sim 1 \mathrm{Gyr}$ (Colberg et al. 2000; Evrard et al. 2002; Cohn \& White 2005). Thus, our survey should identify at least 10 structures such as the Cl 1604 supercluster and detect significant formation activity in the majority of clusters in our sample.

\subsection{The Cluster Sample}

The cluster sample is chosen from various surveys (Oke et al. 1998; Rosati et al. 1998; Vikhlinin et al. 1998; Gladders \& Yee 2000; Gioia et al. 1999, 2003; Stanford et al. 2002; Blanton et al. 2003a; Hashimoto et al. 2005; Henry et al. 2006; Pierre et al. 2006; Maughan et al. 2006) with different detection techniques (optical, X-ray, radio) to reduce any bias associated with sample selection. Table 1 lists the cluster coordinates, redshift, detection technique, availability of high-quality (Chandra and/ or XMM-Newton) X-ray data, status of the optical/near-infrared observations, some notes on the cluster properties, and original references. We note that some of the target clusters, in particular the highest redshift ones, are the targets of wide-field studies by other groups, including the PISCES Survey (Kodama et al. 2005). We have included them in our survey because the number of well-studied clusters at these redshifts is still limited and because there exists a wealth of publicly available ground-based (Subaru and CFHT) and space-based (Hubble Space Telescope (HST), Chandra, XMM-Newton, and/or Spitzer) observations on these systems. Thus, we can increase our sample size at very little observational cost to us.

The cluster sample also includes $\mathrm{Cl} 1604+4304$ at $z=$ 0.90 and $\mathrm{Cl} 1604+4321$ at $z=0.92$, the original clusters detected in the $\mathrm{Cl} 1604$ supercluster (see Gal et al. 2008 and references therein). As the first large-scale structure detected in the ORELSE Survey, the $\mathrm{Cl} 1604$ supercluster currently has the most extensive multiwavelength photometric and multiobject spectroscopic data. As discussed below, we use the properties of the 419 confirmed members in the $\mathrm{Cl} 1604$ supercluster as a guide when choosing the appropriate galaxy magnitude and color selections in our other cluster fields.

\subsection{The Observations}

To detect large-scale structure, we need to probe scales greater than $10 h_{70}^{-1} \mathrm{Mpc}$, the mean projected separation of cluster pairs in local superclusters (Bahcall et al. 1988). Thus, the first phase of our survey is wide-field $\left(25^{\prime}\right)$ optical/nearinfrared $\left(r^{\prime} i^{\prime} z^{\prime} K_{s}\right)$ imaging of the 20 cluster fields. All of the near-infrared observations are original to the ORELSE Survey, while the optical imaging comes from both new and archived observations (see Table 1). For our original observations, we are using the Large Format Camera (LFC) and the Wide-Field Infrared Camera (WIRC) on the Palomar $5 \mathrm{~m}$, Suprime-Cam on the Subaru $8 \mathrm{~m}$, the Wide-Field Infrared Camera (WFCAM) on the UKIRT $3.8 \mathrm{~m}$, and the Florida Multiobject Imaging Near- 
IR Grism Observational Spectrometer (FLAMINGOS) on the KPNO $4 \mathrm{~m}$ to cover an area of at least $0.2 \mathrm{deg}^{2}$ around each target cluster. The optical imaging is initially used to characterize the large-scale environment around the target clusters and choose probable cluster members for follow-up multiobject spectroscopy. The near-infrared $K_{s}$ imaging will be used to measure stellar masses and provide improved photometric redshifts. The photometric observations reach typical depths of $\left\{r^{\prime} i^{\prime} z^{\prime} K_{s}\right\}=\{24.724 .223 .420 .5\}$ for a $3 \sigma$ detection.

Based on the optical $\left(r^{\prime} i^{\prime} z^{\prime}\right)$ imaging for each cluster field, we use broad color-color cuts, characteristic of red, ellipticallike galaxies at the cluster redshift (see Section 4), to produce adaptively smoothed galaxy density maps. These maps are used primarily to provide a visual locator for companion clusters and intermediate-density large-scale structures in the imaging field (see Section 2.4). Filaments and cluster infall regions can cover significant portions of the observed area, but at relatively low contrast, making it difficult to detect them and define their boundaries. Identification of such overdense regions is used to guide the placement of follow-up observations, especially slitmasks for multiobject spectroscopy.

Because photometric techniques for studying galaxy and cluster evolution (such as photometric redshifts, statistical overdensities, and richness estimates) are highly uncertain, the crucial component of this survey, and what distinguishes it from competing studies, is the unprecedented amount of spectroscopic coverage. We utilize the wide-field, multiobject spectroscopic capabilities of the Deep Multiobject Imaging Spectrograph (DEIMOS; Faber et al. 2003) on the Keck $10 \mathrm{~m}$. Because of its large field of view $\left(16.7 \times 5^{\prime} \cdot 0\right)$, capability of positioning up to $120+$ galaxies per slitmask, and high efficiency, DEIMOS is the ideal instrument for a spectroscopic survey of high-redshift large-scale structures. We perform faint object spectroscopy (for objects down to $i^{\prime} \approx 24$ ) with total exposure times of $2.5-3 \mathrm{hr}$. Using the density map as a guide (see above) and a series of appropriate color cuts to preferentially select galaxies at the cluster redshift (see Section 2.3.2), we are obtaining high-resolution $\left(\sim 60 \mathrm{~km} \mathrm{~s}^{-1}\right)$ spectra for $100-200+$ confirmed members per system. Our sample sizes are larger than most of those currently available (e.g., Tanaka et al. 2006, 2007b; Koyama et al. 2007; Swinbank et al. 2007; Fassbender et al. 2008) and allow us to measure properties on a galaxy-togalaxy basis. Based on the spectroscopy completed so far (see Section 3 and Gal et al. 2008), we find that $25 \%-45 \%$ of the galaxies targeted through our system of color selections are at or near the cluster redshift.

Based on our imaging and spectroscopic observations, promising supercluster candidates and other complex systems are currently being targeted for multiwavelength studies with HST, Chandra, Spitzer, and/or the VLA. We note that our large spectroscopic databases are important to capitalize on the multiwavelength data as we can begin to identify and study rare galaxy populations (such as X-ray and radio sources) on an individual rather than a statistical basis (see Kocevski et al. 2009a, 2009b).

\subsection{The Color Selection}

To characterize the large-scale structure around our target clusters, we need to identify galaxies (which will be used to create a density map and which will be targeted for spectroscopy; see below) within the wide-field imaging that are at or near the cluster redshift. To do this, we target red, elliptical-like galaxies which provide the best contrast against the large background

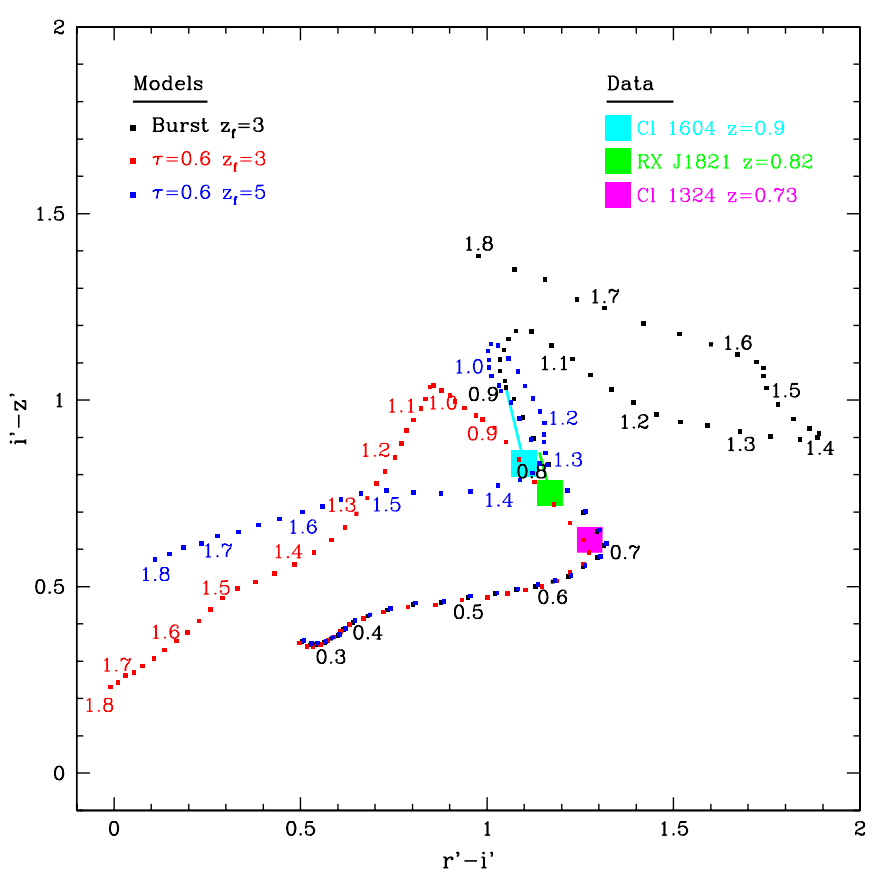

Figure 1. $r^{\prime}-i^{\prime}$ vs. $i^{\prime}-z^{\prime}$ color-color plane. The evolution with redshift for three solar-metallicity BC03 models are shown (see Section 4) as dotted lines with the colors at a given redshift indicated. Large filled squares represent the average colors of spectroscopically confirmed red sequence galaxies in three ORELSE structures, the $\mathrm{Cl} 1604$ supercluster at $z \approx 0.9$ (cyan), the $\mathrm{Cl} 1324$ supercluster at $z \approx 0.73$ (magenta), and RX J1821 at $z=0.82$ (green). Solid lines show the offset from the observed colors of these three systems to the colors predicted by the instantaneous burst model at $z_{f}=3$. No single model fits all three systems.

of blue field galaxies. Until we have sufficient spectroscopy in each field to refine the color selection, we need to choose initial color cuts in the $r^{\prime} i^{\prime} z^{\prime}$ data which are appropriate for the cluster redshift. We have examined the possibility of applying color cuts based on the stellar population synthesis models of Bruzual \& Charlot (2003, hereafter BC03). To see the variation in colors between different star formation histories, we generate synthetic galaxy spectra using three solar-metallicity models : (1) an instantaneous burst with a formation redshift of $z_{f}=3$; (2) a $\tau=0.6 \mathrm{Gyr}$ exponential decline at $z_{f}=3$; and (3) a $\tau=0.6 \mathrm{Gyr}$ exponential decline at $z_{f}=5$. In Gal et al. (2008), we also explored the prescription used by the Redsequence Cluster Survey (RCS, Gladders \& Yee 2005), a model parameterized as a 0.1 Gyr burst ending at $z=2.5$, followed by a $\tau=0.1 \mathrm{Gyr}$ exponential decline. We do not examine the RCS model here as it is nearly identical (to within $0.04 \mathrm{mag}$ ) to the simpler model (1) for the redshift range in which we are interested $(0.6<z<1.3)$.

All three models reproduce the colors of local elliptical galaxies (e.g., Blanton et al. 2003b; Chang et al. 2006) and have formation epochs implied by the observed color evolution in red sequence galaxies (e.g., Stanford et al. 1998; van Dokkum \& Franx 2001; Blakeslee et al. 2006; Mei et al. 2006a; Homeier et al. 2006). The evolution with redshift in the $\left(r^{\prime}-i^{\prime}\right)$ versus $\left(i^{\prime}-z^{\prime}\right)$ color-color diagram (CCD) is shown in Figure 1 for each of the three models. The first thing to note is that all three models are identical at $z \leqslant 0.6$ and nearly identical at redshifts of $0.6<z<1$, suggesting that the color selection at these redshifts is insensitive to the model as long as the formation epoch is sufficiently high. We do, however, observe significant variations at $z>1$, clearly due to differences in formation 
Table 2

The Red Galaxy Color Selection

\begin{tabular}{lcc}
\hline \hline Redshift Range & $r^{\prime}-i^{\prime}$ & $i^{\prime}-z^{\prime}$ \\
\hline $0.6-0.7$ & $1.0-1.4$ & $0.3-0.7$ \\
$0.7-0.8$ & $1.0-1.4$ & $0.4-0.8$ \\
$0.8-0.9$ & $1.0-1.4$ & $0.5-0.9$ \\
$0.9-1.0$ & $1.0-1.4$ & $0.6-1.0$ \\
$>1.0$ & $\cdots$ & $>0.9$ \\
\hline
\end{tabular}

redshift and starburst length. We note that the abrupt changes in color with redshift, most notably in the instantaneous burst model (1), are due to strong Fe II and Mg II absorption lines shifting in and out of the optical bandpasses.

Overlaid in Figure 1 are the average colors of spectroscopically confirmed red sequence galaxies in three ORELSE structures, the $\mathrm{Cl} 1604$ supercluster at $z \approx 0.9$ (Gal et al. 2008), the $\mathrm{Cl} 1324$ supercluster at $z \approx 0.73$ (R. R. Gal et al. 2009 , in preparation), and RX J1821.6+6827 at $z=0.82$ (see Figure 3). We do not include $\mathrm{Cl} 0023$ here as it is dominated by blue galaxies and has a wider red sequence than the other systems (see Section 4.2). This figure demonstrates that a single model does not fit all three systems. In particular, the colors from the $\mathrm{Cl} 1604$ supercluster and RX J1821.6+6827 are redder in $r^{\prime}-i^{\prime}$ and bluer in $i^{\prime}-z^{\prime}$ than all of the models. Such discrepancies are unsurprising as the evolutionary history of these systems are undoubtedly different and more complex. In addition, a galaxy's star formation history will vary with environment. As a result, we adopt broad $(\Delta m=0.4)$ color cuts that were used successfully in the $\mathrm{Cl} 1604$ supercluster. We use the models as a guide and offset our color cuts relative to those used in $\mathrm{Cl} 1604$ (see Section 2.2 of Gal et al. 2008). For cluster redshifts between $z=0.6$ and 1.0, we use a standard $r^{\prime}-i^{\prime}$ cut of $1-1.4$ but vary the $i^{\prime}-z^{\prime}$ cut depending on the cluster redshift. As done for the $\mathrm{Cl} 1604$ supercluster, we adopt an $i^{\prime}-z^{\prime}$ cut of $0.6-1.0$ for a cluster at $z=0.9-1.0$. For those clusters at lower redshifts, we adopt an $i^{\prime}-z^{\prime}$ cut which gets progressively bluer by $0.1 \mathrm{mag}$ as the redshift interval decreases by $\Delta z=0.1$. For cluster redshifts of $z>1$, we use a fixed cut of $i^{\prime}-z^{\prime}>0.9$. We use a single color cut at these redshifts because, while the $r^{\prime}-i^{\prime}$ color at $z>1$ is highly dependent on the model, the $i^{\prime}-z^{\prime}$ colors are consistently above $\sim 0.9$, except for models with recent or continuing star formation. We note that ACS observations of three X-ray-selected clusters at $z>1$ all reveal well-defined red sequences with characteristic colors of $i_{\mathrm{F} 775 \mathrm{~W}}-z_{\mathrm{F} 850 \mathrm{LP}} \approx 1$ (Mei et al. 2006a, 2006b; Demarco et al. 2007). These results are completely consistent with our adopted $i^{\prime}-z^{\prime}$ color cut. Table 2 lists the exact color cuts used in this survey.

We note that our red galaxy selection plus density-mapping technique is fundamentally different from cluster-detection methods such as the red-sequence (RCS) method and MaxBCG (Koester et al. 2007). We use a simple, relatively wide $(\Delta m \geqslant$ $0.4 \mathrm{mag}$ ) color cut to examine the projected density of objects with colors broadly consistent with those of red galaxies at the redshift of interest. RCS and MaxBCG both rely on the presence of a tight red sequence (with an assumed intrinsic scatter of 0.075 and 0.060 , respectively), which, especially at higher redshift, may impose a priori requirements on the types of systems detected. In the ORELSE Survey, we target fields with known structures and search for lower significance density enhancements, such as groups and filaments, whose constituent galaxies, while more evolved (redder) on average than field galaxies, do not necessarily form a tight red sequence (as is the case for $\mathrm{Cl}$ 0023; see Section 4.2). While we have made broad assumptions on the formation history of the cluster galaxies when choosing our color cuts (particularly at $z>1$ ) in order to eliminate fore/background galaxies, the redshifts of our targets are already known, so we do not require a photometric redshift estimator such as the red-sequence fitting (which relies on one particular evolutionary model) used by RCS.

\subsubsection{Magnitude Limits for the Density Map}

In addition to the color cuts used to select red galaxies at the cluster redshift, we also need to define the range of $i^{\prime}$ magnitudes used to produce the density map (see below). In the case of the Cl 1604 supercluster at $z=0.9$, we chose $i^{\prime}=20.5-23.5$ based on our existing spectroscopy. For consistency, we shift the $i^{\prime}$ magnitude limits to the redshift of the other target clusters by using $k$-corrections derived from the instantaneous burst at $z_{f}=3$ model (1) shown in Figure 1. The magnitude limits used to create the red galaxy density maps for the two target clusters presented in this paper are given in Section 3.3.

\subsubsection{Priorities for Spectroscopic Observations}

When determining which galaxies to target for multiobject spectroscopy, we prioritize galaxies based on color. As a first priority, we select objects which meet our color cut(s) for red galaxies described above. Within this priority (and the other lower priorities), we weight objects by their $i^{\prime}$-band flux with preference given to brighter objects. We include objects with $i^{\prime}$ between the minimum magnitude used to create the density map (see Section 2.3.1) and $i^{\prime}=24$, the limit where we can still obtain reasonably high signal-to-noise ratio $(\sim 1-5)$ spectra in our 2.5-3 hr exposures. With increasingly lower priority, we select galaxies which are progressively bluer in both $r^{\prime}-i^{\prime}$ and $i^{\prime}-z^{\prime}$ to target galaxies within the green valley and into the blue cloud. Although our contamination rates are higher for bluer galaxies, we observe a significant population of blue, starforming members in the $\mathrm{Cl} 1604$ supercluster (see Figure 1 of Gal et al. 2008) and $\mathrm{Cl} 0023$ (see Figure 2), so it is essential to target these galaxies as well. We note that we always maximize the number of slits per slitmask (given our minimum slit length of $5^{\prime \prime}$ ) by including all other galaxies, within our magnitude limits, at the lowest priority. The placement of these slitmasks (with the prioritized galaxy selection described above) on the sky is guided by the structure (i.e., overdense regions and filaments) identified in the red galaxy density map (see Section 2.4).

\subsection{Producing the Density Map}

Following Gal \& Lubin (2004), we produce a red galaxy density map for each cluster field by adaptively smoothing a subset of galaxies which satisfy the galaxy color selection and magnitude limits determined in Section 2.3. An adaptive kernel smoothing is applied using an initial window of $0.75 h_{70}^{-1}$ $\mathrm{Mpc}$ and $10^{\prime \prime}$ pixels. The chosen kernel size prevents small groups from being blended into single detections in the density map and enhances the contrast of small groups against the background, making detection of such low-mass systems easier. We stress that the density maps of color-color-selected galaxies are primarily a tool for the visual identification of structures such as clusters, groups, walls, and filaments.

\subsubsection{The Cluster-Detection Threshold}

Because the ORELSE Survey aims to detect comparatively low mass systems, our cluster/group selection algorithm is 

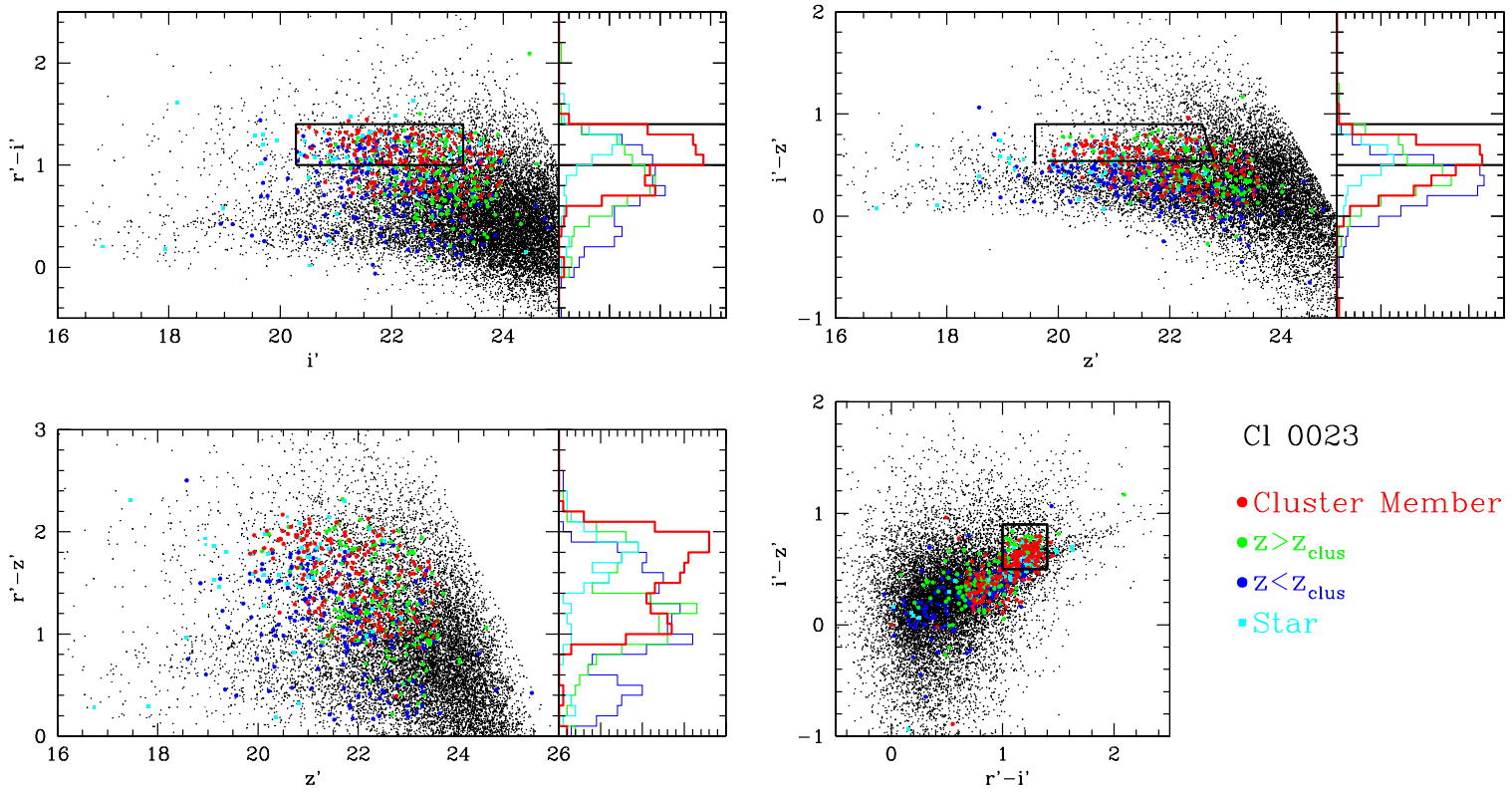

Cl 0023

- Cluster Member

- $\mathrm{z}>\mathrm{z}_{\text {clus }}$

- $\mathrm{z}<\mathrm{z}_{\text {clus }}$

- Star

Figure 2. $i^{\prime}$ vs. $\left(r^{\prime}-i^{\prime}\right), z^{\prime}$ vs. $\left(r^{\prime}-z^{\prime}\right), z^{\prime}$ vs. $\left(i^{\prime}-z^{\prime}\right)$, and $\left(r^{\prime}-i^{\prime}\right)$ vs. $\left(i^{\prime}-z^{\prime}\right)$ CMDs and CCDs of the Cl 0023 field. All objects in the LFC imaging are shown as small dots. The black rectangular regions outline the color selections applied to produce the density maps and prioritize spectroscopic targets. Spectroscopically confirmed system members are overplotted as large red dots. Foreground galaxies at $z<0.82$ are shown as blue dots, background galaxies at $z>0.87$ are green dots, and cyan dots are stars. Color distributions of spectroscopic objects are shown to the right of each CMD.
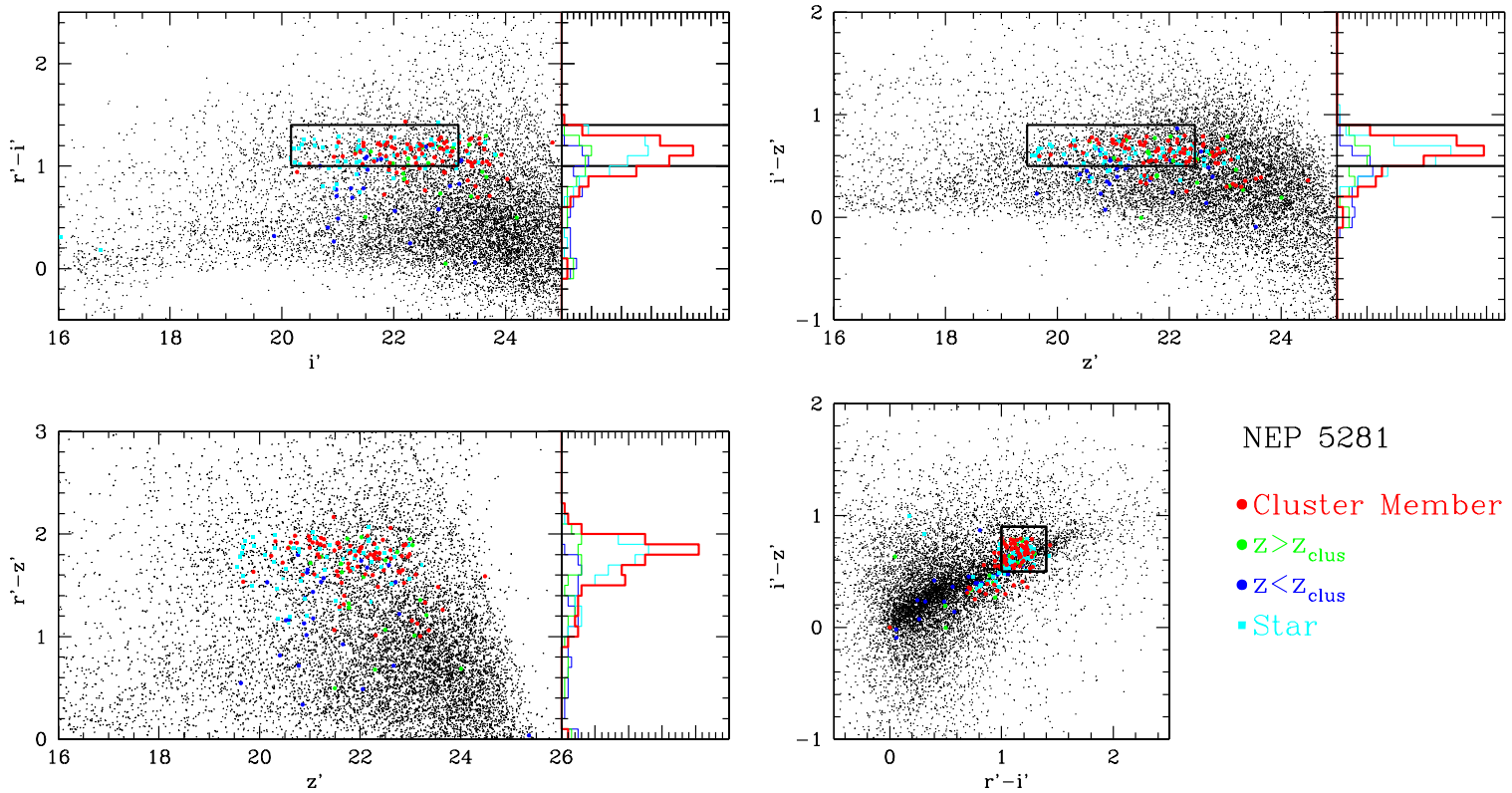

NEP 5281

- Cluster Member

$\cdot \mathrm{z}>\mathrm{z}_{\text {clus }}$

$\cdot \mathrm{z}<\mathrm{z}_{\text {clus }}$

"Star

Figure 3. Same as Figure 2 but for the RX J1821 field. Spectroscopically confirmed cluster members are overplotted as large red dots. Foreground galaxies at $z<0.80$ are shown as blue dots, background galaxies at $z>0.84$ are green dots, and cyan dots are stars.

potentially susceptible to a high false detection rate. It is, therefore, useful to select overdensities on the basis of some objective criteria. The application of both $r^{\prime}-i^{\prime}$ and $i^{\prime}-z^{\prime}$ color cuts to select galaxies for the density maps results in a nontrivial relationship between projected galaxy density and the cluster mass. A complete treatment of selection functions would require large cosmological simulations with model galaxy colors in our photometric system and would still depend on the assumed cosmology and recipes for modeling galaxy formation. Therefore, we rely instead on two observational datasets: the extensive photometry and spectroscopy in the field of the Cl 1604 supercluster at $z=0.9$ (Gal \& Lubin 2004; Gal et al. 2008) for the cluster component and the Canada-France-
Hawaii Telescope Legacy Survey (CFHTLS) Deep Fields ${ }^{4}$ for the "field" component.

The $\mathrm{Cl} 1604$ system consists of numerous groups and clusters, with velocity dispersions as low as $\sim 300 \mathrm{~km} \mathrm{~s}^{-1}$, corresponding to a mass of $\sim 10^{14} h_{70}^{-1} M_{\odot}$. The spectroscopic catalog of over 400 confirmed supercluster members allows us to reliably

\footnotetext{
4 Based on observations obtained with MegaPrime/MegaCam, a joint project of CFHT and CEA/DAPNIA, at the Canada-France-Hawaii Telescope (CFHT) which is operated by the National Research Council (NRC) of Canada, the Institut National des Science de l'Univers of the Centre National de la Recherche Scientifique (CNRS) of France, and the University of Hawaii. This work is based in part on data products produced at TERAPIX and the Canadian Astronomy Data Centre as part of the CFHTLS, a collaborative project of NRC and CNRS
} 
identify such low mass systems and examine their properties in the map of galaxy density. We use the structure identified as Cluster C in Gal et al. (2008) to set our detection thresholds because it is the lowest-mass group to be spectroscopically confirmed in the $\mathrm{Cl} 1604$ supercluster. This poor cluster is evident as a single peak in the density map and has a sufficient number of spectroscopic members (18 within $\left.1 h_{70}^{-1} \mathrm{Mpc}\right)$ to estimate its velocity dispersion $\sigma_{C}=313 \mathrm{~km} \mathrm{~s}^{-1}$. Following Gal \& Lubin (2004), we use SExtractor to detect structures in the density maps. The SExtractor parameters must be chosen with care, to recover systems which we know to be real (in this case, Cluster $\mathrm{C}$ and all of the more massive systems in $\mathrm{Cl} 1604$ ), while minimizing the number of spurious detections.

To measure the false detection rate, we require a galaxy distribution that retains the general correlation properties of galaxies meeting our color-color selection criteria. Optimally, we would measure the correlation function of field galaxies from the same data used for cluster selection. However, our imaging survey is entirely targeted at known high-density regions of the universe. Thus, we use the CFHTLS to derive the statistical properties of the red galaxy distribution. The 2008A data release includes four fields, of $\sim 2 \mathrm{deg}^{2}$ each, at widely separately locations on the sky. Having four distinct fields allows us to estimate not only the typical false detection rate but also the range due to cosmic variance. Matched photometric catalogs for all four fields are provided, including $r^{\prime} i^{\prime} z^{\prime}$ photometry (the same as our data) in the AB system. In brief, following Postman et al. (1996) and Gal \& Lubin (2004), we use the CFHTLS data to compute Raleigh-Levy (RL) parameters for galaxies meeting our color and magnitude cuts which are then used to generate simulated galaxy distributions. These distributions are used to produce density maps, on which we run SExtractor. The SExtractor parameters DETECT_THRESH and MIN_AREA are varied until a set of values is found that successfully detects

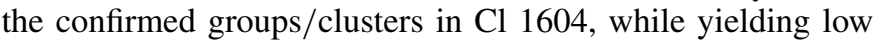
contamination.

In each CFHTLS Deep Field, we select those objects classified as galaxies, meeting the same quality, $i^{\prime}$ magnitude, and $r^{\prime}-i^{\prime}, i^{\prime}-z^{\prime}$ cuts applied to the Palomar LFC data for the $\mathrm{Cl} 1604$ imaging. This gives the number of galaxies $N_{\mathrm{RL}, f}$ that should be used in the RL simulation for the $f$ th CFHTLS Deep Field. This number varies for every ORELSE target as the magnitude and color limits change. A square region of exactly $1^{\circ}$ on a side is selected from the center of each CFHTLS Deep Field to simplify computation of the RL parameters and avoid underexposed field edges. We then generate a set of simulated RL distributions over a grid of values for the two RL parameters $\theta_{0}$ and $d$. The best match to the observed CFHTLS data yields values of $\theta_{0}=100^{\prime \prime}, d=0.6$ using the color and magnitude cuts from $\mathrm{Cl} 1604$. These parameters are comparable to those found by Gal \& Lubin (2004) and Lopes et al. (2006).

The next step is to create 100 simulated galaxy distributions for each Deep Field using these RL parameters, each simulation having $N_{\mathrm{RL}, f}$ galaxies. This yields 400 total density maps. We then run the same adaptive kernel density mapper applied to the real data on these simulated distributions and detect overdensities using SExtractor. We vary the DETECT_THRESH and DETECT_MINAREA parameters, using values of the galaxy density at different radii $r_{\text {test }}$ from the center of Cluster $\mathrm{C}$ in $\mathrm{Cl}$ 1604 , setting DETECT_MINAREA to $\pi r_{\text {test }}^{2}$. We find that an area of $47 \mathrm{pixel}^{2}$, corresponding to a radius of $0.3 h_{70}^{-1} \mathrm{Mpc}$, allows us to detect all of the spectroscopically confirmed cluster candidates in the real data, while minimizing the false candi- dates detected in the RL simulations. The corresponding galaxy density threshold is 7300 galaxies per square degree. The median number of false detections expected in the area imaged for $\mathrm{Cl} 1604$ is $N_{\text {false }}=0.6$, compared to the 10 candidates detected (see Figures 2-3 of Gal et al. 2008). The range of false detections based on the four CFHTLS Deep Fields is between 0.3 and 0.9 for the $\mathrm{Cl} 1604$ area.

As demonstrated in Lopes et al. (2006), the RL simulations provide a good estimate of the false detection rate. A much simpler approach is to generate purely random galaxy distributions, but these do not encode any of the galaxy correlations present in the real universe. Nevertheless, they serve as a useful confirmation of the RL results. We performed the same analysis described above, using random positions instead of an RL distribution. As expected, we detect many fewer false candidates, with $N_{\text {false }}=0.4$ for the $\mathrm{Cl} 1604$ area.

\subsubsection{False Detection Rates}

Having set the SExtractor detection parameters based on $\mathrm{Cl} 1604$, we run cluster detection on the density maps for other cluster fields with the same parameters. However, because the color and magnitude limits vary from field to field based on the cluster redshifts and pre-existing spectroscopy, the RL parameters and especially the number of galaxies used in the RL simulation for each field will vary, resulting in different contamination rates. First, we followed the same procedure described above to determine the optimal RL parameters for each ORELSE targets. For all such targets, we find that the range of well-matched parameters is quite broad, so we fix the RL parameters to $\theta_{0}=100^{\prime \prime}, d=0.6$ as found for $\mathrm{Cl} 1604$. Therefore, the only quantity that we change in our RL simulation from target to target is the total number of galaxies.

To determine the number of galaxies $N_{\mathrm{RL}}$ to use for each ORELSE target, we simply apply the $i^{\prime}$ magnitude limits and $r^{\prime}-i^{\prime}$ and $i^{\prime}-z^{\prime}$ color cuts used for the target to each of the four CFHTLS Deep Fields.

For every target field $t$, we proceed as follows.

1. We generate $100 \mathrm{RL}$ distributions over each square CFHTLS Deep Field with $N_{\mathrm{RL}, t}$ galaxies. This number changes between the Deep Fields due to cosmic variance.

2. Density maps are created from each of the distributions, with the same $0.75 h_{70}^{-1} \mathrm{Mpc}$ smoothing used for the corresponding target.

3. SExtractor is run with DETECT_MINAREA corresponding to a radius of $0.3 h_{70}^{-1} \mathrm{Mpc}$ at the redshift $z_{t}$ of the target, and DETECT_THRESH fixed at the galaxy density from Cluster $\mathrm{C}$ in $\mathrm{Cl} 1604$.

4. The number of expected false detections $N_{\text {false, } t}$ is set to the mean value from the 400 simulations, scaled to the area imaged for target $t$.

The median number of false detections (and their range from the four CHFTLS Deep Fields) expected in the fields of the two target clusters discussed in this paper, $\mathrm{Cl} 0023$ at $z=0.84$ and RX J1821.6 at $z=0.82$, is $0.60(0.3-1.0)$ and $0.55(0.3-$ 0.8 ), respectively. Cosmic variance on the scale of the LFC field could still potentially cause significant changes in the number of false detections. We, therefore, repeated the above process, now using 100 randomly placed LFC-sized subfields within each of the 400 RL density maps for each target. We find that the median and rms number of false detections is $0.5 \pm 0.8$ and $0.3 \pm 0.6$ for $\mathrm{Cl} 0023$ and RX J1821, respectively. However, the median 
richnesses of these false detections is typically much lower than for the overdensities found in our target fields (see Section 4).

\section{THE TARGETS AND OBSERVATIONS}

\section{1. $\mathrm{Cl} 0023+0423$}

$\mathrm{Cl} 0023$ at $z=0.84$ was originally detected as an overdensity in the distant cluster survey of Gunn et al. (1986) which covered $71.5 \mathrm{deg}^{2}$ using photographic plates taken with the Palomar $1.2 \mathrm{~m}$ and $5 \mathrm{~m}$ and the KPNO $4 \mathrm{~m}$ telescopes. This system was later included in the Oke et al. (1998) survey using the Low-Resolution Imaging Spectrograph (LRIS; Oke et al. 1995) on the Keck $10 \mathrm{~m}$. This study spectroscopically confirmed 24 members and revealed that this system is actually comprised of two small groups, with velocity dispersions of 158 and $415 \mathrm{~km} \mathrm{~s}^{-1}$ and separated by $2922 \mathrm{~km} \mathrm{~s}^{-1}$ in radial velocity (Postman et al. 1998). $N$-body simulations implied that the two groups were likely bound, were currently merging, and would form a massive cluster $\left(\sigma_{\text {los }} \approx 730 \mathrm{~km} \mathrm{~s}^{-1}\right)$ in the next $\sim 1$ Gyr (Lubin et al. 1998b). Based on the LRIS photometry and spectroscopy, Postman et al. (1998) found that the population is quite active, with $57 \%$ of the confirmed members showing [O II] emission with equivalent widths (EWs) greater than $15 \AA$ and the majority with broad-band spectral energy distributions (color ages) which suggest star formation within the past $3 \mathrm{Gyr}$. Twelve confirmed members were covered by a single WFPC2 (F702W) image centered on this system. $75 \%$ are late-type galaxies, a morphological fraction more consistent with galaxy groups or the field than rich clusters at these redshifts (Lubin et al. 1998a).

Follow-up multiwavelength studies of this system also indicate substantial star formation and AGN activity. SCUBA observations of four high-redshift $(0.84 \leqslant z \leqslant 1.27)$ clusters, including $\mathrm{Cl}$ 0023, reveal 10 securely identified submillimeter sources detected toward the cluster fields, a combined source excess at $850 \mu \mathrm{m}$ of $\sim 3-4$ times blank field surveys (Best 2002). Similarly, an $\mathrm{H}_{\alpha}$ imaging survey of $\mathrm{Cl} 0023$ revealed a high integrated SFR per cluster mass of $(58.4 \pm 5.2) h^{-3} M_{\odot}$ $\mathrm{yr}^{-1} / 10^{14} M_{\odot}, 10$ times higher than that observed in $\mathrm{H}_{\alpha}$ cluster surveys at $z \approx 0.2$ (Finn et al. 2004). Finn et al. (2004) also do not find a population of early-type galaxies in $\mathrm{Cl} 0023$ with ongoing star formation (i.e., detectable $\mathrm{H}_{\alpha}$ emission), suggesting that the $[\mathrm{O} \mathrm{II}]$ emission that we observe in otherwise red, passive galaxies may, in fact, be due to the AGN activity (see Section 7).

\section{2. $R X J 1821.6+6827$}

RX J1821 at $z=0.82$ was the highest redshift cluster discovered in the ROSAT North Ecliptic Pole (NEP) survey (Gioia et al. 2003; Henry et al. 2006). XMM-Newton observations revealed slightly elongated $\mathrm{X}$-ray emission, with a bolometric luminosity of $1.17_{-0.18}^{+0.13} \times 10^{45} h_{70}^{-2} \mathrm{erg} \mathrm{s}^{-1}$ and a temperature of $4.7_{-0.7}^{+1.2} \mathrm{keV}$ (Gioia et al. 2004). Spectroscopic redshifts for 20 cluster galaxies using the Keck I telescope and the CFHT imply a cluster velocity dispersion of $775_{-113}^{+182} \mathrm{~km} \mathrm{~s}^{-1}$, typical of a relatively rich cluster (Gioia et al. 2004).

The cores of all 18 clusters in the NEP survey were imaged by the VLA at $1.4 \mathrm{GHz}$ (Branchesi et al. 2006). A total of 32 radio sources, with peak brightnesses $\geqslant 0.17$ mJy beam $^{-1}$, were detected, suggesting a $3 \sigma$ overdensity of sources relative to the field within $0.4 h_{70}^{-1} \mathrm{Mpc}$. One of these sources was a confirmed member of RX J1821. These results indicate radio
AGN activity in the cluster cores, consistent with studies of other clusters (Owen et al. 1999; Best 2003; Best et al. 2002; Miller \& Owen 2003).

\subsection{Photometric Data}

Cl 0023 and RX J1821 were imaged using the LFC (Simcoe et al. 2000) on the Palomar $5 \mathrm{~m}$ telescope. The LFC is a mosaic of six $2048 \times 4096$ CCDs in a cross-shaped layout, mounted at prime focus. The imaging area corresponds roughly to the unvignetted region of a circle $24^{\prime}$ in diameter. Data were taken on UT 2004 April 27 and 2004 August 15, with the camera installed in the standard North-South orientation and all six CCDs in use. The imaging data were taken in unbinned mode, with a pixel scale of $0^{\prime \prime} .182$ pixel $^{-1}$. Average seeing was $1^{\prime \prime} .0$, and the nights were not photometric. Individual exposures of $450 \mathrm{~s}$ were taken, moving the telescope by up to $45^{\prime \prime}$ in each direction to alleviate the gaps due to the spaces between the CCDs. Each target was imaged in the SDSS $r^{\prime} i^{\prime} z^{\prime}$ filters, with total exposure times in these filters of $\{450036002250\} \mathrm{s}$ for $\mathrm{Cl}$ 0023 and $\{630040503600\}$ s for RX J1821.

Data reduction was performed using the IRAF data-reduction suite (Tody 1986), including the external package MSCRED. We followed the general mosaic imaging guidelines used by the NOAO Deep Wide-Field Survey (Jannuzi et al. 2004), with modifications appropriate to our data. The details of the data reduction are described in Gal et al. (2005). Object detection in the target frames was performed by running SExtractor in dual-image mode, using ultra-deep images (combining all three bands) for detection, while measurements were performed on the single-band images. Elliptical photometry apertures are determined from the deep image, and these same apertures are used for the individual filter images. This procedure results in catalogs where magnitudes are measured using identical apertures in all three filters, which improves the measurement of galaxy colors by using the same physical size for each galaxy in all filters (Lubin et al. 2000). Due to the lack of calibration, we assumed photometric zero points, color terms, and atmospheric extinction terms from other photometric nights, resulting in semicalibrated catalogs.

To provide photometric calibration, RX J1821 was observed with the Orthogonal Parallel Transfer Imaging Camera (OPTIC; Howell \& Tonry 2003) installed on the University of Hawai' $i$ $2.2 \mathrm{~m}$ telescope on UT 2007 July 22. This instrument contains two $2 \mathrm{k} \times 4 \mathrm{k}$ CCDs mounted side-by-side, with 0'. 14 pixels. While this instrument is capable of orthogonal transfer charge shifting to improve image quality, we used it in conventional mode to minimize data reduction complexity. Three exposures of $300 \mathrm{~s}$ each were obtained in each of the SDSS $r^{\prime}, i^{\prime}$, and $z^{\prime}$ filters in photometric conditions with $0^{\prime \prime} .8$ seeing. Numerous SDSS standard stars were also observed to derive photometric calibration. These observations were processed and sources detected the same way as for our LFC data. The calibrated, photometric source catalogs from OPTIC were matched to the semicalibrated catalogs from LFC, and transformations of the form

$$
m_{\text {calib }}=m_{\mathrm{LFC}}+A \times(\text { color })+B
$$

were derived for each filter, using the $r^{\prime}-i^{\prime}$ color for the $r^{\prime}$ and $i^{\prime}$ filters, and $i^{\prime}-z^{\prime}$ color for $z^{\prime}$. These transformations were then applied to the full source catalogs from the LFC data. Unfortunately, we have not yet been able to obtain photometric calibration for the $\mathrm{Cl} 0023$ field. Comparison of the semicalibrated FC data of RX J1821 with the final calibration 

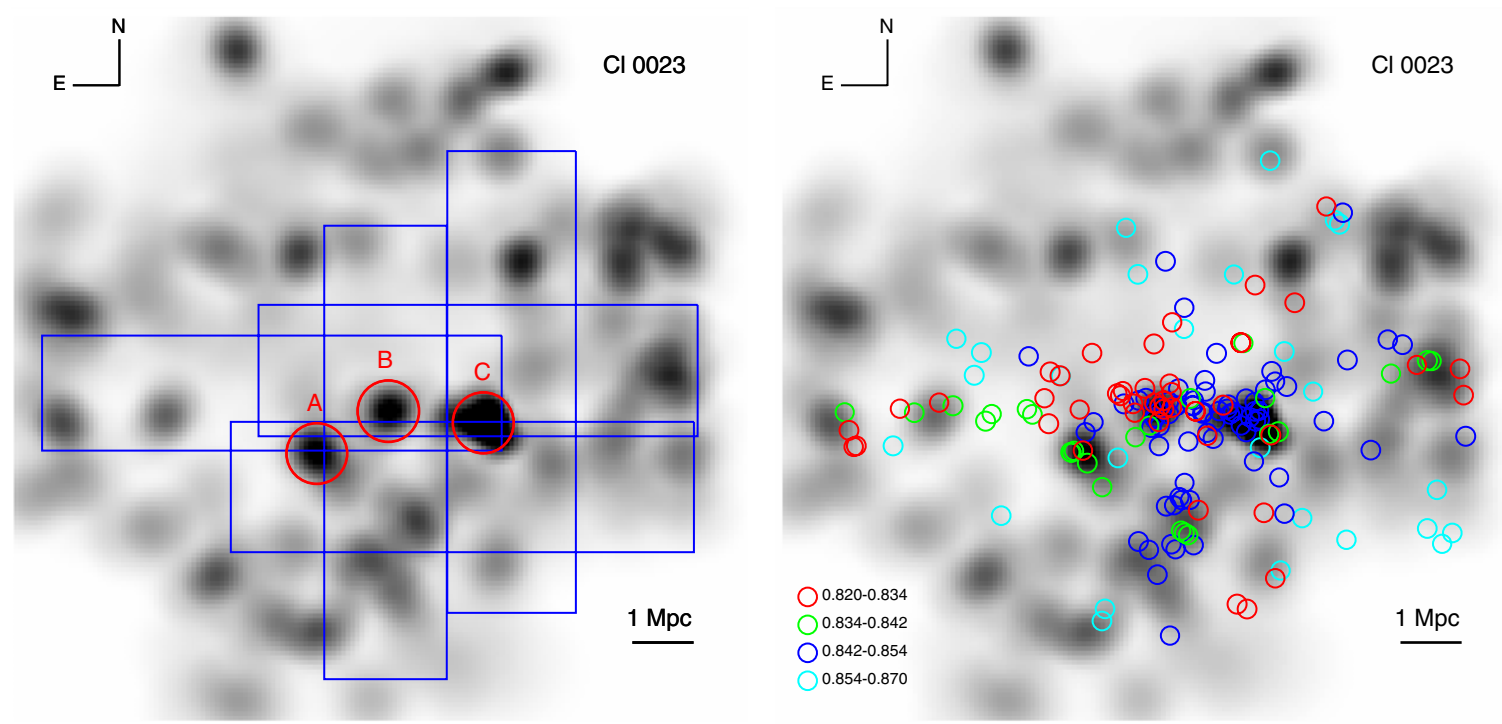

Figure 4. Adaptive kernel density map of color-selected red galaxies in the $\mathrm{Cl} 0023$ field. Left: candidate groups/clusters, which meet our detection threshold (see Section 2.4), are marked with red circles of $0.5 h_{70}^{-1} \mathrm{Mpc}$ and labeled with an identifying letter. Large blue rectangles indicate the layout of the five DEIMOS masks. Right: small circles indicate the 169 confirmed members, coded by color representing four redshift ranges which correspond to peaks in the redshift distribution in Figure 6. The distribution of confirmed members corresponds extremely well to peaks in the density map, including, as expected, the three significant peaks but also the lower density peak directly South of component B.
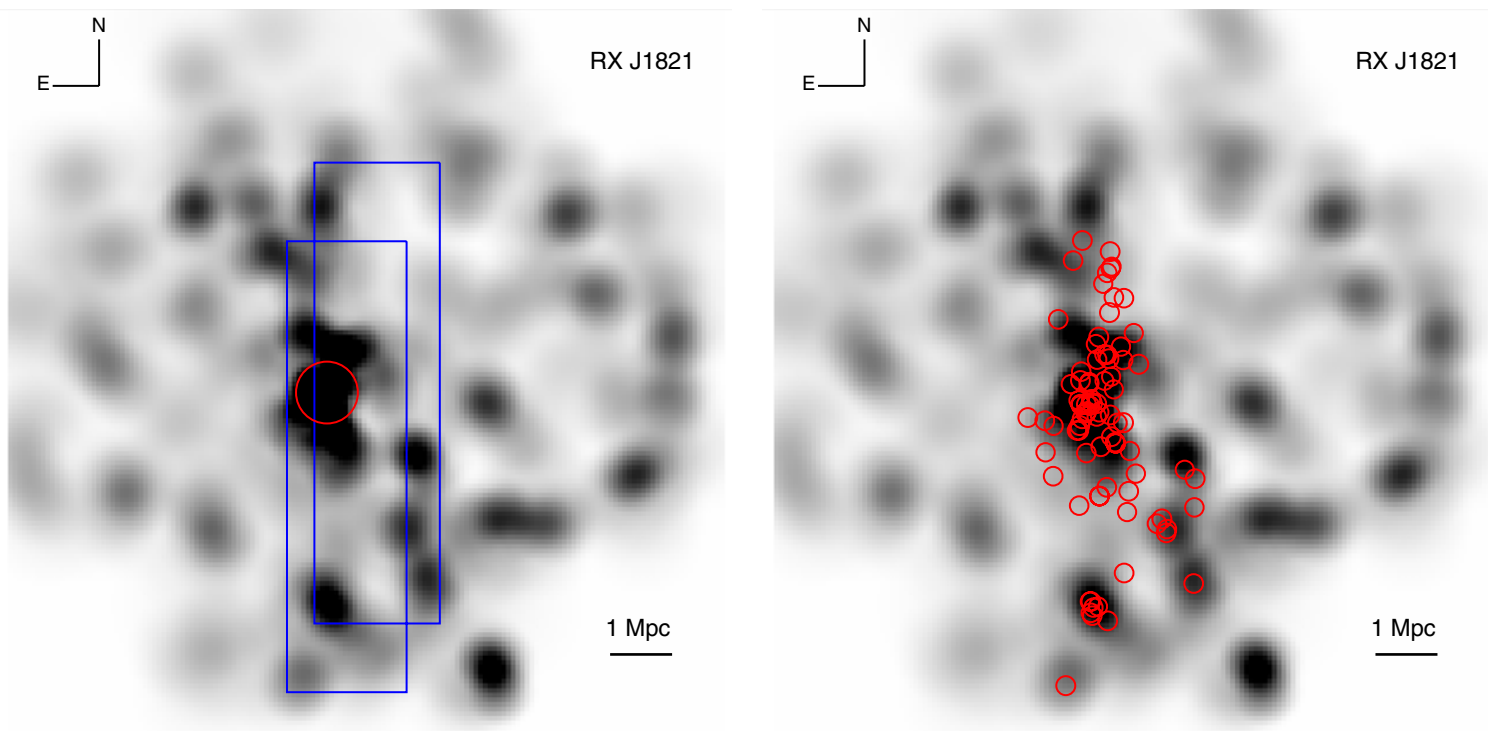

Figure 5. Adaptive kernel density map of color-selected red galaxies in the RX J1821 field. Left: only the cluster itself, marked by a red circle of radius $0.5 h_{70}^{-1} \mathrm{Mpc}$, meets our detection threshold. Large blue rectangles indicate the layout of the two DEIMOS masks. Right: small red circles indicate the 85 confirmed cluster members. As in Figure 4, the distribution of confirmed members follows extremely well the overdense regions in the density map, tracing the elongated structure of the cluster and even picking up the lower density peaks (small groups) to the South and southwest.

using OPTIC suggests that zero points in each filter may be incorrect by $0.05-0.1 \mathrm{mag}$, with errors of $\sim 0.1$ on the color term.

The resulting color-magnitude diagrams (CMDs) and CCDs for the $\mathrm{Cl} 0023$ and RX J1821 fields are shown in Figures 2 and 3 . Based on the photometry described above, red galaxy density maps were made using the appropriate color-color cuts (see Section 4 and Table 2) and $i^{\prime}$-band magnitude ranges of 20.2823.28 and 20.16-23.16 for $\mathrm{Cl} 0023$ and RX J1821, respectively (see Section 2.3.1). Figures 4 and 5 show the resulting density maps. Using the RL simulation of Section 2.4, we expect 0.60 and 0.55 false detections in the $\mathrm{Cl} 0023$ and RX J1821 fields, respectively, significantly fewer the actual number that we detect (see Section 4).

\subsection{Spectroscopic Data}

Both $\mathrm{Cl} 0023$ and RX J1821 were observed with the DEIMOS (Faber et al. 2003) on the Keck $10 \mathrm{~m}$ telescopes. We used the $12001 \mathrm{~mm}^{-1}$ grating, blazed at $7500 \AA$, with the OG550 filter and $1^{\prime \prime}$ slits. This setup results in a pixel scale of $0.33 \AA$ pixel $^{-1}$, a resolution of $\sim 1.7 \AA\left(68 \mathrm{~km} \mathrm{~s}^{-1}\right)$, and typical spectral coverage from $6250 \AA$ to $8900 \AA$. Galaxies as faint as $i^{\prime} \approx 24$ were observed, with highest priority given to red galaxies based on the color cuts used to produce the initial density maps from the semicalibrated photometry. Approximately $60 \%$ of the targeted objects met the red galaxy color cut. Lower priorities were given to galaxies increasingly blueward (in $r^{\prime}-i^{\prime}$ and/or $i^{\prime}-z^{\prime}$ ) of the highest priority box. $\mathrm{Cl} 0023$ was observed with three masks 
and RX J1821 with two masks on UT 2005 September 2-3 in 0.7 seeing, with five exposures of $1800 \mathrm{~s}$ each on each mask. $\mathrm{Cl} 0023$ was observed with two additional masks on UT 2007 September 12 with five exposures of $1800 \mathrm{~s}$ each in 0.8 seeing and thin cirrus.

Data were reduced using the DEEP2 version of the spec $2 d$ and specld data reduction pipelines (Davis et al. 2003). Redshifts were obtained using DEEP2's redshift measurement pipeline zspec (M. C. Cooper et al. 2009, in preparation), with typical redshift errors of $25 \mathrm{~km} \mathrm{~s}^{-1}$. Each redshift was assigned a quality flag between 1 and 4, where 1 indicates a secure redshift could not be determined due to poor signal, lack of features, or reduction artifacts; 2 is a redshift obtained from either a single feature or two marginally detected features; 3 is a redshift derived from at least one secure and one marginal feature; and 4 is assigned to spectra with redshifts obtained from several high signal-to-noise features. A quality of -1 is used for sources securely identified as stars. Qualities of 3 or 4 indicate secure extragalactic redshifts and only those are used in the following analyses (see also Gal et al. 2008).

In the $\mathrm{Cl} 0023$ field, we obtained 423 extragalactic redshifts with $Q \geqslant 3$, of which 54 were serendipitous detections. An additional 73 galaxy redshifts were incorporated from the spectroscopic survey of Oke et al. (1998). The combined catalog contains $Q \geqslant 3$ redshifts for 169 structure members with $0.82<z<0.87$. In the RX J1821 field, a total of 129 extragalactic redshifts with $Q \geqslant 3$ were obtained in the RX J1821 field, 26 of which were serendipitous detections. Thirteen additional redshifts were taken from Gioia et al. (2004). The combined catalog contains $Q \geqslant 3$ redshifts for 85 structure members with $0.80<z<0.84$.

\section{THE RESULTS}

\subsection{The Global Properties}

In Figures 4 and 5, we show the red galaxy density maps for the $\mathrm{Cl} 0023$ and RX J1821 fields. The density peaks which meet our detection threshold (see Section 2.4.1) are circled. We find three peaks in the $\mathrm{Cl} 0023$ field and one in the $\mathrm{RX}$ J1821 field, all significant based on our estimated false detection rate. The observed structures in these maps are consistent with that expected from the redshift distributions shown in Figures 6 and 7. While Postman et al. (1998) found only two peaks in the redshift distribution, we see four clear peaks with our more extensive spectroscopy. Combined with the density map, these results suggest an even more complex system of groups. In contrast, the redshift histogram of RX J1821 shows a single peak at $z \approx 0.82$ (albeit with some clear velocity substructure; see below) which is consistent with the single, massive overdensity observed in the density map.

In addition, the distribution of confirmed members in each system follows exceedingly well both the peaks and lower density regions in the two density maps. In the $\mathrm{Cl} 0023$ field, although many of the confirmed members are spread out across the spectroscopic area, 54\% coincide directly (within $1 h_{70}^{-1}$ $\mathrm{Mpc}$ ) with the three significant peaks (labeled A, B, and C). This correlation is not due to spectroscopic coverage alone as the five DEIMOS masks cover a large fraction of the imaging area, with most of that area covered by at most two masks (see the left panel of Figure 4). We also see that the density map does an excellent job at picking up associated lower density structures, such as the less-significant peak directly to the South of component B. Splitting the confirmed members by redshift according to the four redshift peaks in Figure 6, we find velocity segregation between the three components. Component $\mathrm{A}$ is largely comprised of galaxies belonging to the redshift peak at $z \approx 0.839$, while component $\mathrm{C}$ is largely comprised of galaxies belonging to the redshift peak at $z \approx 0.845$. Component $\mathrm{B}$, however, is actually a superposition of two groups along the lineof-sight, one which corresponds to the lowest redshift peak at $z \approx 0.828$ and the other which corresponds to the redshift peak at $z \approx 0.845$, same as component $\mathrm{C}$. The galaxies within the highest redshift peak at $z \approx 0.864$ are not centrally concentrated but rather extend across the entire region, suggesting a sheet of galaxies in the near background.

In Figure 8, we show the redshift histograms, within radii of 0.5 and $1.0 h_{70}^{-1} \mathrm{Mpc}$, centered on the three overdense components. Following the procedure described in Gal et al. (2008), we use these distributions to measure the biweight velocity dispersion computed by ROSTAT (Beers et al. 1990). Components $\mathrm{A}$ and $\mathrm{C}$ represent single systems with measured velocity dispersions between 428 and $497 \mathrm{~km} \mathrm{~s}^{-1}$, typical of massive groups or poor clusters (e.g., Zabludoff \& Mulchaey 1998). Component B is comprised of two smaller galaxy groups with measured velocity dispersion of 206-293 $\mathrm{km} \mathrm{s}^{-1}$ (see Table 3). As such, $\mathrm{Cl} 0023$ is a four-way groupgroup merger. Based on virial mass estimates of the individual groups, the final mass of the merged cluster will be $\sim 5 \times 10^{14} h_{70}^{-1} M_{\odot}$, typical of a rich cluster (e.g., Girardi et al. 1998).

While $\mathrm{Cl} 0023$ is dynamically active, RX J1821 is considerably more relaxed with a single peak in both the density map and redshift histogram (see Figures 5 and 7); however, there are indications of continuing formation in this cluster as well. In particular, the overdense region in the density map corresponding to the cluster is clearly extended in the North-South direction,

Table 3

Target Coordinates, Redshifts, and Velocity Dispersions

\begin{tabular}{|c|c|c|c|c|c|c|c|c|c|}
\hline \multirow[b]{2}{*}{ Name } & \multirow[b]{2}{*}{ ID } & \multicolumn{2}{|c|}{$\mathrm{J} 2000.0$} & \multicolumn{3}{|c|}{ Within $0.5 h_{70}^{-1} \mathrm{Mpc}$} & \multicolumn{3}{|c|}{ Within $1.0 h_{70}^{-1} \mathrm{Mpc}$} \\
\hline & & R.A. & Decl. & $N$ & $z_{\text {mean }}$ & $\sigma$ & $N$ & $z_{\mathrm{med}}$ & $\sigma$ \\
\hline \multirow[t]{4}{*}{$\mathrm{Cl} 0023+0423$} & A & 6.0223 & 4.3586 & 5 & 0.8383 & $\ldots{ }^{a}$ & 7 & 0.8390 & $479 \pm 172$ \\
\hline & $\mathrm{B} 1^{\mathrm{b}}$ & 5.9764 & 4.3874 & 10 & 0.8286 & $242 \pm 54$ & 17 & 0.8282 & $206 \pm 32$ \\
\hline & $\mathrm{B} 2^{\mathrm{b}}$ & 5.9671 & 4.3824 & 12 & 0.8445 & $177 \pm 118$ & 27 & 0.8453 & $293 \pm 79$ \\
\hline & $\mathrm{C}$ & 5.9227 & 4.3824 & 15 & 0.8451 & $548 \pm 84$ & 29 & 0.8463 & $428 \pm 67$ \\
\hline RX J1821.6+6827 & & 275.3801 & 68.4651 & 17 & 0.8170 & $993 \pm 87$ & 40 & 0.8193 & $926 \pm 77$ \\
\hline
\end{tabular}

Notes.

${ }^{a}$ Insufficient redshifts to compute dispersion.

${ }^{\mathrm{b}}$ There are two distinct velocity peaks in this component (see Figure 8). 

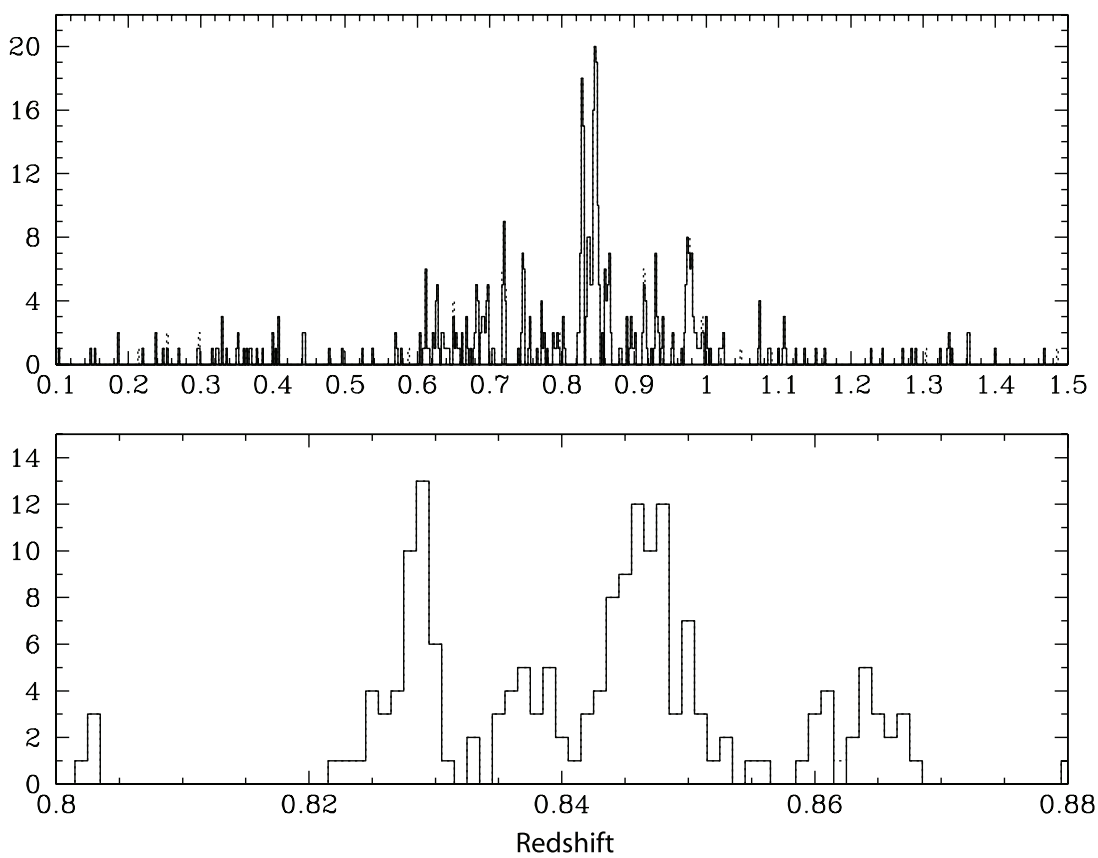

Figure 6. Redshift distribution in the $\mathrm{Cl} 0023$ field. The top panel shows all extragalactic objects (dotted line) and only those with high redshift quality (solid line). The bottom panel focuses on the redshift range of the group-group merger with redshift bins of $\Delta z=0.001$. There are four clear peaks in the redshift histogram, indicative of the dynamical state of the system.
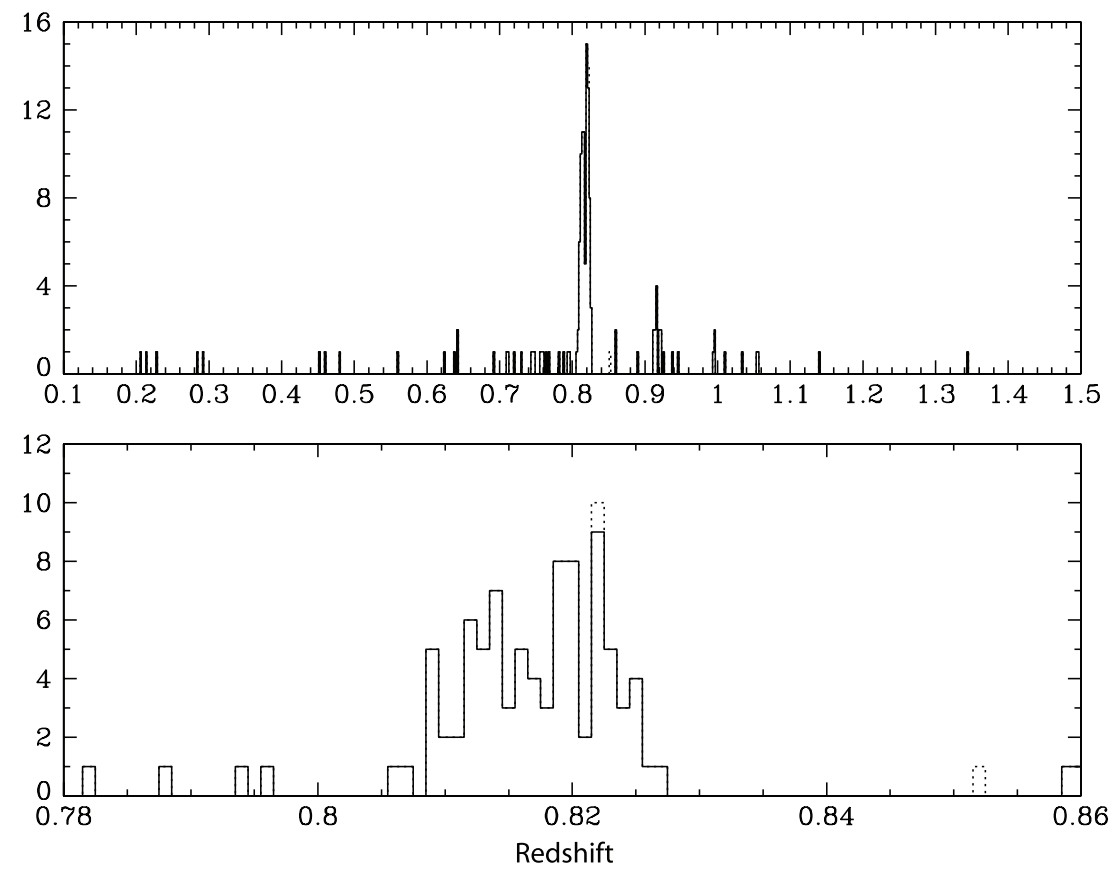

Figure 7. Same as Figure 6 for the RX J1821 field. There is a single clear peak $z \approx 0.817$ which is completely isolated in redshift space; however, the distribution shows clear indications of velocity substructure (see also Figure 9).

consistent with the X-ray contours from Gioia et al. (2004). As in $\mathrm{Cl} \mathrm{0023,} \mathrm{the} \mathrm{distribution} \mathrm{of} \mathrm{confirmed} \mathrm{members} \mathrm{follows} \mathrm{ex-}$ tremely well the overdense regions in the density map, tracing the elongated structure of the cluster and even picking up the lower density peaks (small groups) to the South and southwest which are kinematically associated with the cluster (right panel of Figure 5).

To determine if RX J1821 has any velocity segregation in the plane of the sky, we have binned the velocities by sky position going from North to South along the elongated structure. We use four bins, each with an equal number of galaxies.
Comparing each resulting velocity histogram to the full velocity histogram using the KS test, only the southernmost distribution is marginally different, with a $6 \%$ probability of being drawn from the same distribution, possible due to an infalling group (see Figure 5). Otherwise, no statistically significant velocity segregation was detected.

Velocity histograms, centered on the density peak and measured within a projected radius of $0.5,1.0$, and $1.5 h_{70}^{-1} \mathrm{Mpc}$, are shown in Figure 9. We measure biweight velocity dispersions between 926 and $993 \mathrm{~km} \mathrm{~s}^{-1}$ (see Table 3). We note that three ROSTAT dispersion estimators (sigma, biweight, and 

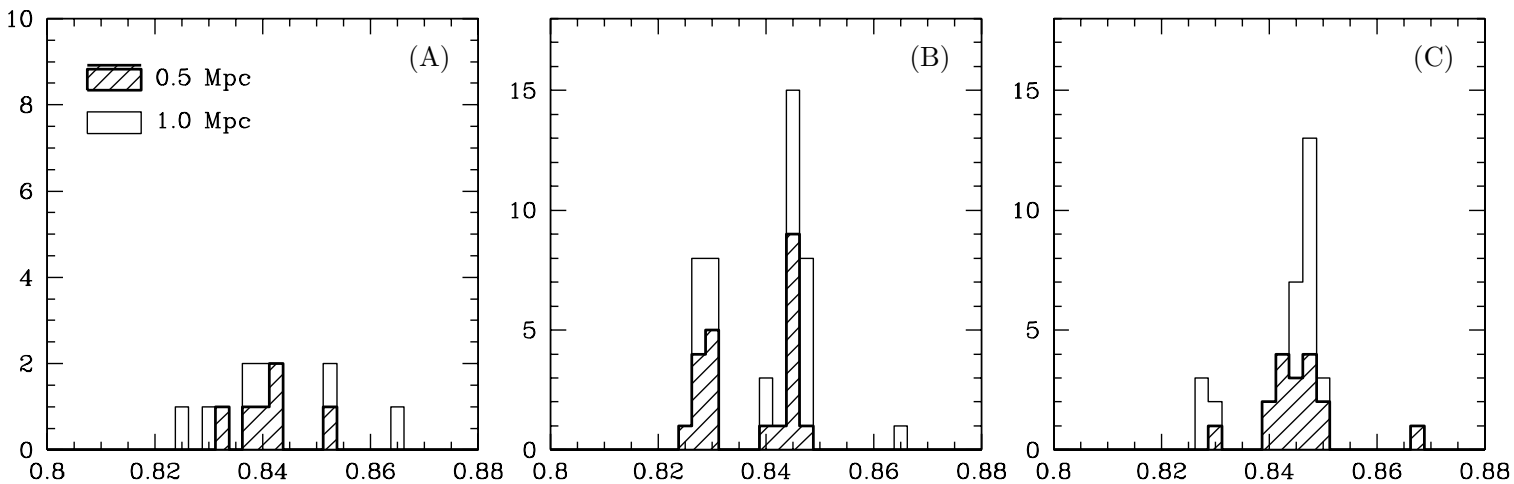

Figure 8. Redshift histograms for components $\mathrm{A}, \mathrm{B}$, and $\mathrm{C}$ identified in the density map of the $\mathrm{Cl} 0023$ field (see Figure 4 ). The shaded regions show the distribution within a projected radius of $0.5 h_{70}^{-1} \mathrm{Mpc}$, while the solid line corresponds to a radius of $1 h_{70}^{-1} \mathrm{Mpc}$. Components A and C have reasonably well-defined redshift peaks with measured velocity dispersions that are consistent with galaxy groups (see Table 3), while component B is actually a superposition of two distinct groups along the line of sight.
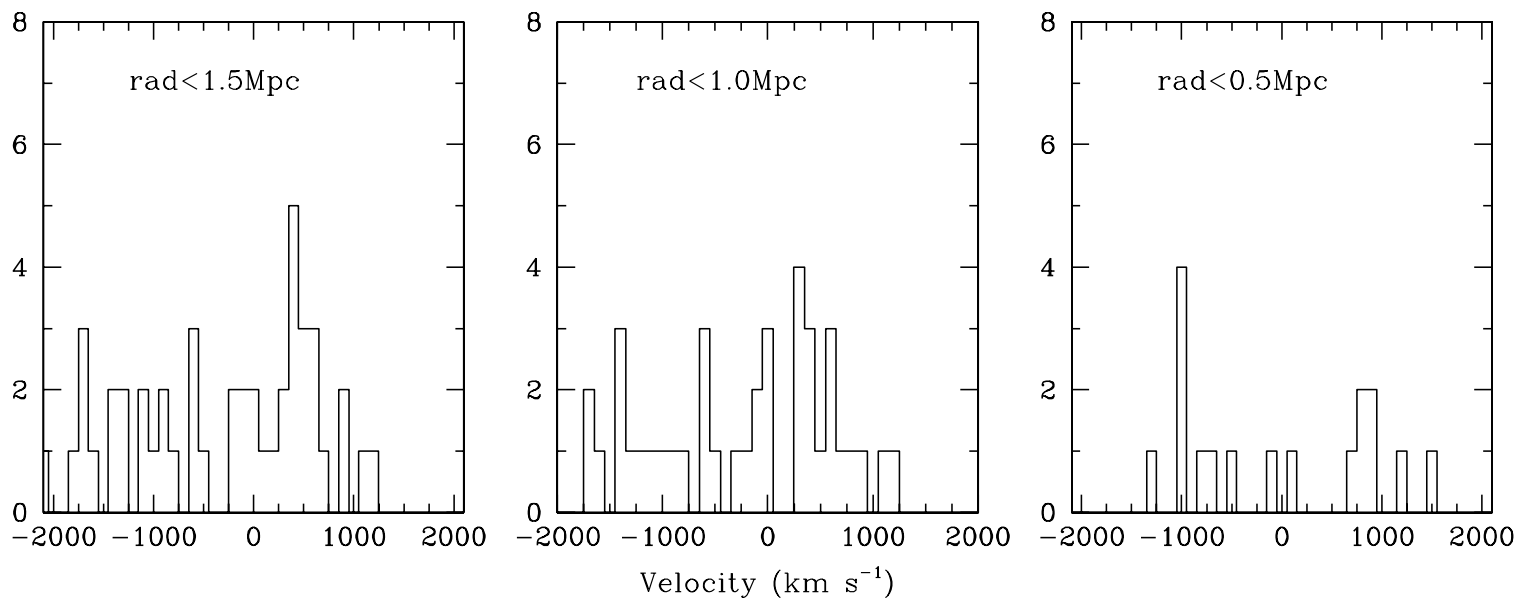

Figure 9. Velocity histograms of the cluster RX J1821 within projected radii of $0.5,1.0$, and $1.5 h_{70}^{-1} \mathrm{Mpc}$. Each velocity is plotted relative to the median velocity of the member galaxies in each bin. Although the velocity dispersion estimators are quite consistent within a radial bin and from bin to bin, the distributions are clearly non-Gaussian. The cluster does not, however, have clear multiple components such as $\mathrm{Cl} 0023$ or a significant amount of nearby structure such as the $\mathrm{Cl} 1604$ supercluster.
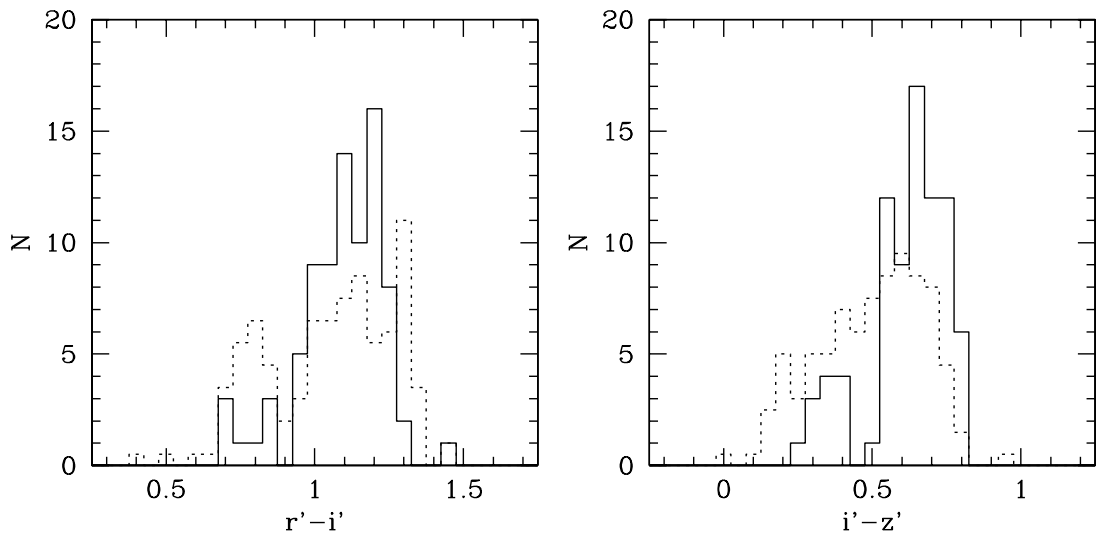

Figure 10. Histogram of $r^{\prime}-i^{\prime}$ (left panel) and $i^{\prime}-z^{\prime}$ (right panel) colors of confirmed member galaxies (down to $\left.i^{\prime}=24\right)$ in $\mathrm{Cl} 0023$ (dotted histogram) and $\mathrm{RX}$ $\mathrm{J} 1821$ (solid histogram). RX J1821 shows a tighter red-sequence and a smaller percentage of blue galaxies than $\mathrm{Cl} 0023$.

gapper) give consistent measurements within a radial bin and from bin to bin; however, as is clear from Figure 9, the velocity distributions are clearly non-Gaussian, implying measurable velocity substructure. RX J1821 does not, however, have clear multiple components such as $\mathrm{Cl} 0023$ or a significant amount of nearby structure such as the $\mathrm{Cl} 1604$ supercluster (Gal et al. 2008).
The variation in system properties might reflect the differences in selection technique. Based on the handful of ORELSE targets observed so far, we find that optical selection appears to preferentially select systems which are dynamically active $(\mathrm{Cl}$ 0023), are embedded in complex, large-scale structures (the $\mathrm{Cl}$ 1324 and 1604 superclusters), contain lower mass constituents (all of the above), and have less (or no) diffuse gas emission (all of the above; see Lubin et al. 2004; Kocevski et al. 2009a; 
Kocevski et al. 2009c). As often suggested, X-ray selection appears to detect more dynamically evolved, concentrated, and isolated clusters (RX J1821 and another NEP cluster, RX $\mathrm{J} 1757.3+6631$ at $z=0.691)$ at these redshifts.

\subsection{The Galaxy Populations}

Although $\mathrm{Cl} 0023$ and RX J1821 are at almost identical redshifts, they are distinctly different in their evolutionary state. As a four-way group merger, $\mathrm{Cl} 0023$ is at the very early stage of hierarchical cluster formation. In comparison, the cluster RX J1821 has progressed further along its dynamical evolutionary track as evidenced by its more concentrated velocity and spatial structure. These global differences translate to substantial differences in their galaxy populations as well. Based on the CMDs in Figures 2-3 and the color histograms in Figure 10, Cl 0023 has a significantly larger population of blue galaxies, with $51 \%$ being bluer than the red galaxy color-color cut (down to $\left.i^{\prime}=24.5\right)$, compared to only $24 \%$ in RX J1821. RX J1821 also has a tight, well-defined red-sequence which extends down to at least $i^{\prime} \simeq 23.5$, unlike the wider one of $\mathrm{Cl} 0023$ (although its larger width may be due, in part, to photometric errors; see Section 3.3). The CMD of RX J1821 indicates a population of both old, massive (bright) red galaxies with high formation epochs $\left(z_{f} \sim 2-3\right)$, consistent with previous studies of massive clusters (e.g., Mei et al. 2006a and references therein), as well as quenched galaxies as evidenced by the presence of faint red sequence galaxies (e.g., Koyama et al. 2007 and references herein) and stronger $\mathrm{H} \delta$ absorption (see below).

The diversity in activity is also evident in the galaxy spectra. In $\mathrm{Cl} 0023,80 \%$ of the confirmed members have detectable [O II] emission (EWs greater than $2 \AA$ ), including detectable line emission in otherwise red, passive galaxies (with $\mathrm{Ca} \mathrm{H} \& \mathrm{~K}$ but no Balmer absorption). We note that some of the [O II] emission, in particular in these passive galaxies, may actually be due to AGN and/or extended emission-line regions, i.e., Seyferts or LINERs (e.g., Yan et al. 2006). This high [O II] fraction is consistent with the results of Poggianti et al. (2006) who studied a sample of groups and clusters at $z=0.4-0.8$ from the ESO Distant Cluster Survey (EDisCS). They find an anticorrelation between [O II] fraction $\left(f_{[\mathrm{OII}]}\right)$ and velocity dispersion $(\sigma)$, with the majority of groups $\left(\sigma<500 \mathrm{~km} \mathrm{~s}^{-1}\right)$ having $f_{[\mathrm{OII}]} \gtrsim 0.7$ (see Figure 4 of Poggianti et al. 2006). Thus, the high [O II] fraction in $\mathrm{Cl}$ 0023 could just result from the fact that the constituents of the system are all group-sized. In contrast, the population of the massive cluster RX J1821 is more quiescent, with only $36 \%$ of the galaxies showing [O $\mathrm{II}]$ emission. The [O II] fraction is completely consistent with that found in EDisCS clusters with similar velocity dispersions. Although comparable to other moderate-to-high redshift clusters, we note that the measured fraction in RX J1821 is higher than the $f_{[\mathrm{OII}]} \approx 0.23$ found in nearby $(z=0.04-0.08)$ SDSS clusters with similar dispersions (see Figure 6 of Poggianti et al. 2006), consistent with the increase in blue, star-forming cluster galaxies with redshift (e.g., Butcher \& Oemler 1984; Ellingson et al. 2001).

In Figure 11, we show a comparison between the composite spectra from the $\mathrm{Cl} 0023$ and RX J1821 galaxy populations. When making the composites, we use only the DEIMOS spectra because of their higher quality and spectral resolution. Each spectrum is normalized (by their average rest-frame 3700-4300 $\AA$ flux) and weighted by its $i^{\prime}$-band luminosity. A small fraction (less than 13\%) of galaxies are excluded because of instrumental effects or bad columns in their spectra or nondetections in the photometry. The resulting composites include 138 and 69

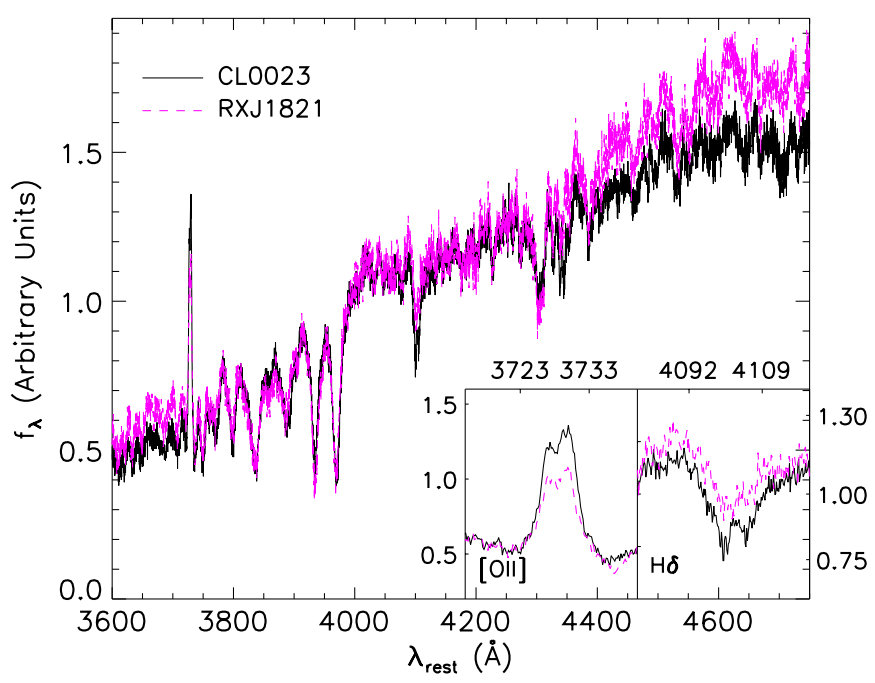

Figure 11. Comparison between the composite DEIMOS spectra of $\mathrm{Cl} 0023$ (solid black line) and RX J1821 (dashed magenta line). Only confirmed members with redshift quality $Q \geqslant 3$ are used in both cases: 138 and 69 members for $\mathrm{Cl} 0023$ and RX J1821, respectively (see Section 7). The two composites are normalized so that their fluxes match at $4050 \AA$. Insets in the lower right show close ups of the $[\mathrm{O}$ II] and $\mathrm{H} \delta$ lines. As already indicated by their colors, the galaxy population of $\mathrm{Cl} 0023$ is considerably more active than that of RX J1821.

(A color version of this figure is available in the online journal.)

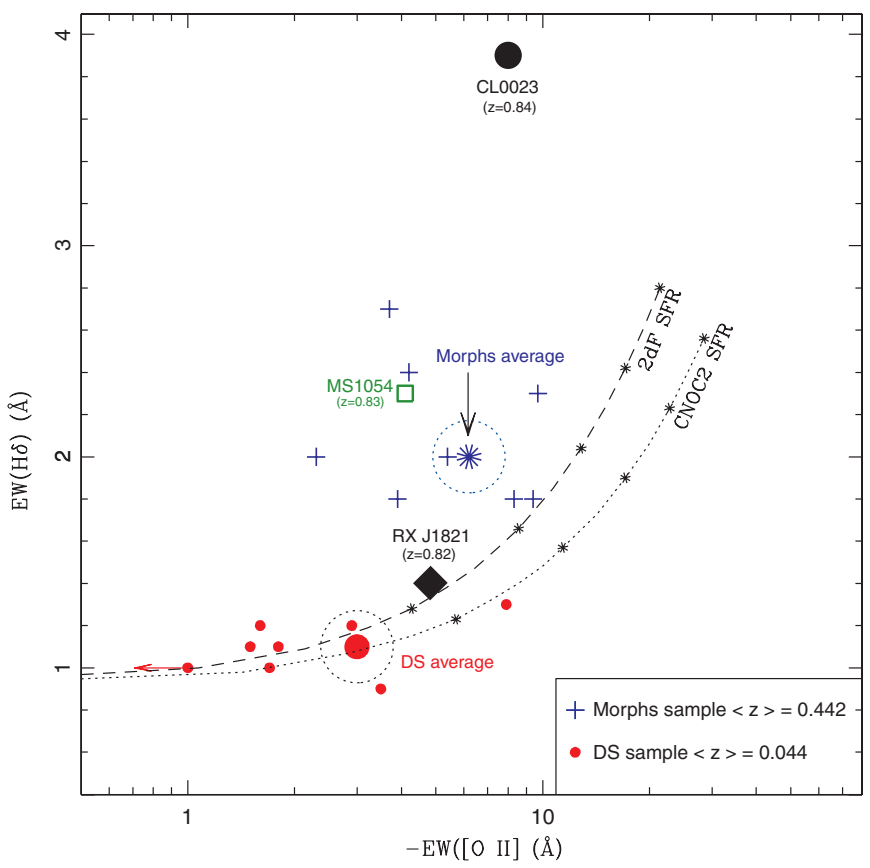

Figure 12. Adapted Dressler et al. (2004) plot of [O II] vs. H $\delta$ EW. The two dashed lines (based on the CNOC2 and $2 \mathrm{dF}$ surveys) indicate the average spectral properties of a galaxy population consisting of passive galaxies plus a varying fraction of continuously star-forming galaxies. The small asterisks along the curves mark the appropriate values of $\mathrm{EW}([\mathrm{O} \mathrm{II}])$ and $\mathrm{EW}(\mathrm{H} \delta)$ for $20 \%, 40 \%$, $60 \%, 80 \%$, and $100 \%$ mix of galaxies undergoing continuous star formation. Local clusters fall on the lines, while moderate-redshift clusters, as does the massive cluster MS 1054 at $z=0.83$ (open green square), lie above indicating some contribution from galaxies that have undergone a recent starburst. The average properties of the $\mathrm{Cl} 0023$ system (large black circle) is substantially offset in both [O $\mathrm{II}]$ and $\mathrm{H} \delta$, implying a high fraction of star-forming galaxies and an even larger contribution from recent starbursts. In contrast, the X-rayselected, isolated cluster RX J1821 at $z=0.82$ (large black diamond) has more moderate $[\mathrm{O} \mathrm{II}]$ emission and falls close to the normal star formation lines, indicating a large quiescent galaxy population (as also evident in the CMD of Figure 3) and only a modest starburst contribution.

(A color version of this figure is available in the online journal.) 
galaxies in $\mathrm{Cl} 0023$ and $\mathrm{RX} \mathrm{J1821,} \mathrm{respectively.} \mathrm{The} \mathrm{comparison}$ reveals clear differences between the two galaxy populations. The $\mathrm{Cl} 0023$ composite has stronger [O II] and $\mathrm{H} \delta$ lines, as expected from the overall blue colors of the galaxy population. There is also evidence of significant infill of the $\mathrm{H} \delta$ line from ongoing star formation which is essentially absent in the RX $\mathrm{J} 1821$ data. Without this infill (or equivalently $\sim 100 \mathrm{Myr}$ after star formation is truncated), the apparent contribution from $\mathrm{A}$ stars (i.e., the poststarburst mode) would be much higher than for RX J1821. Conversely, the composite of RX J1821 has strong $\mathrm{Ca} H \& \mathrm{~K}$ lines and a significant excess of flux at wavelengths greater than $4500 \AA$, showing a stronger contribution from $\mathrm{K}$ and $\mathrm{M}$ stars. Using the standard bandpasses from Fisher et al. (1998), we measure the EWs of the [O II] and $\mathrm{H} \delta$ line for each composite spectrum. We find $\{\mathrm{OII}, \mathrm{H} \delta\}=\{-8.0,3.9\}$ for $\mathrm{Cl}$ 0023 and $\{-4.7,1.4\}$ for RX J1821. Using slightly different bandpasses (from, e.g., Postman et al. 1998; Balogh et al. 1999) or the line-fitting functionality of the IRAF ${ }^{2}$ task splot changes the EWs by less than $20 \%$ for $\mathrm{H} \delta$ and less than $10 \%$ for [O II].

Because the spectroscopy in $\mathrm{Cl} 0023$ is more extensive than that in RX J1821 (i.e., five versus two slitmasks, respectively), it is possible that we probe further into the bluer, low spectroscopic priority population, thus skewing the comparison of the two systems. To test this possibility, we compared only the data from the first two slitmasks for each of the two fields. In these subsamples, $\mathrm{Cl} 0023$ has 85 confirmed members, 54 of which (64\%) meet our red galaxy color cut, whereas RX J1821 has 69 confirmed members, with $56(81 \%)$ meeting this color cut. These percentages, along with Figures 2 and 3, confirm the higher red galaxy fraction in RX J1821. The excess red galaxies in RX J1821 are faint, red sequence galaxies which are much less common in $\mathrm{Cl} \mathrm{0023,} \mathrm{where} \mathrm{we} \mathrm{instead} \mathrm{find} \mathrm{more} \mathrm{luminous}$ blue galaxies. The EWs of the [O II] and $\mathrm{H} \delta$ lines in the $\mathrm{Cl} 0023$ composite are essentially unchanged from the full sample. Even if we consider only the high-priority (red) galaxy populations from the first two masks, we find $\{\mathrm{OII}, \mathrm{H} \delta\}=\{-5.8,3.4\}$ for $\mathrm{Cl} 0023$ and $\{-2.9,1.0\}$ for RX J1821. As expected, the overall line strengths are weaker in the red galaxies than in the full sample, but the differences between the two clusters persist. Even the red galaxy population in $\mathrm{Cl} 0023$ is more active in star-forming/AGN activity than it is in RX J1821.

In Figure 12, we place the results of our composite spectra (from the total galaxy samples) on the Dressler et al. (2004) plot of [O II] versus $\mathrm{H} \delta$ EW. The two dashed lines, based on the CNOC2 and $2 \mathrm{dF}$ field surveys, indicate the average spectral properties of a galaxy population which consists of passive galaxies plus a varying fraction of continuously starforming galaxies. The low-redshift $2 \mathrm{dF}$ data represent the low SFR case, while the moderate-redshift $(z \sim 0.4)$ CNOC2 data represents the high SFR case. The small asterisks on the curved lines indicate subsequently higher fractions of normal star-forming galaxies, ranging from $20 \%$ to $100 \%$ of the total cluster population. Local clusters from the Dressler \& Shectman (1988; hereafter DS) sample fall directly on the lines, indicating a cluster population largely dominated by quiescent galaxies plus a small contribution of continuously star-forming galaxies. Moderate-redshift $(\langle z\rangle=0.442)$ clusters from the Morphs sample (Dressler et al. 1999), as well as the massive cluster MS 1054 at $z=0.83$ (van Dokkum et al. 2000), all have average $\mathrm{H} \delta$ strengths of $\sim 2 \AA$ and have substantial [O $\mathrm{II}$ ] emission

\footnotetext{
5 IRAF is distributed by the National Optical Astronomy Observatory, which is operated by the Association of Universities for Research in Astronomy, Inc., under cooperative agreement with the National Science Foundation.
}

ranging from -3 to $-10 \AA$. Both features indicate significantly more ongoing star formation than found in the local sample (see Dressler et al. 2004). Unlike the nearby clusters, the moderateredshift clusters lie above the lines, implying an $\mathrm{H} \delta$ line which is too strong to be produced by normal star formation. These results indicate a larger contribution from galaxies that have undergone a recent starburst.

When placed on this diagram, we see that the average properties of the $\mathrm{Cl} 0023$ population is on the high end of the $[\mathrm{O}$ II] distribution from the Morphs sample and, more significantly, substantially offset in $\mathrm{H} \delta$, above both the normal star-forming lines as well as the Morphs average. These results imply an even larger contribution from recent starbursts in the $\mathrm{Cl} 0023$ system. In contrast, the RX J1821 population has a weaker [O II] line, nearer to the low end of the Morphs sample, and falls considerably closer to the normal star-forming lines. These results indicate a large quiescent galaxy population, a small population of continuously star-forming galaxies (as also evident in the CMD of Figure 3), and only a modest contribution from starburst galaxies. As noted by Dressler et al. (2004), there is strong evolution in the [O II] and $\mathrm{H} \delta$ features from low to moderate redshift. However, there is no obvious trend to higher redshifts. At $z \approx 0.4-0.9$, the structures all occupy regions of phase space which corresponds to activity levels higher than local clusters. In all cases, they lie above the normal star formation lines but with large cluster-to-cluster variations, indicating different evolutionary histories and, subsequently, different starburst contributions.

As a caveat, we note that an exact comparison of $\mathrm{Cl} 0023$ and RX J1821 to other surveys depends on the spectroscopic selection criteria. We have shown above that we can directly compare the populations of $\mathrm{Cl} 0023$ and RX J1821. However, the DS and Morphs spectroscopic samples are derived largely (although not entirely; see Dressler et al. 1999) from magnitudelimited surveys. Because red (presumably less active) galaxies are given a higher priority in our spectroscopic survey, the resulting composite spectra of $\mathrm{Cl} 0023$ and RX J1821 may be biased, relative to the DS and Morphs samples, toward smaller [O II] and $\mathrm{H} \delta$ EWs. As such, our measurements would represent lower limits on the star formation activity. In the case of $\mathrm{Cl} 0023$, correcting this bias would likely increase its already substantial offset from the lower redshift samples and the normal star formation lines, further confirming the extreme level of activity in this system.

To quantify this bias, we plan to measure the average (and range) of spectral properties (e.g., EWs) as a function of color and magnitude once the spectroscopic data are completed for a significant portion of our survey. Incompleteness, especially in the faint blue population, can be measured by statistically subtracting the typical field contribution in any given color and magnitude range using deep imaging taken with the same filters as part of the CFHTLS. Combining the two, we can estimate (and account for) the effect of bias on the cluster properties, in particular the blue fractions and composite spectra. In addition, we will use photometric redshifts where possible to more accurately determine our spectroscopic selection function. However, accurate photometric redshifts require mid-infrared data in the IRAC bands which are currently only available for the $\mathrm{Cl} 1604$ supercluster field.

\section{CONCLUSIONS}

In this paper, we have presented the motivation, design, and implementation of the ORELSE Survey, a systematic 
search for structure on scales of $10 h_{70}^{-1} \mathrm{Mpc}$ around 20 well-known clusters at $0.6<z<1.3$. The survey covers $5 \mathrm{deg}^{2}$, all targeted at intermediate-to-high-density regions, making it complementary and comparable to field surveys such as DEEP2 and COSMOS. The program utilizes optical/nearinfrared imaging at the UKIRT $3.8 \mathrm{~m}$, KPNO $4 \mathrm{~m}$, Palomar $5 \mathrm{~m}$, and Subaru $8 \mathrm{~m}$ covering at least $25^{\prime}$ around each target cluster. Following the successful application in the $\mathrm{Cl} 1604$ supercluster at $z \approx 0.9$, we use an adaptively smoothed red galaxy density map to visually identify associated groups/clusters and larger scale filaments/walls. Guided largely by actual observations, we use the extensive sample of 400+ confirmed members in $\mathrm{Cl} 1604$ supercluster to adapt our color and magnitude cuts, quantify significant density peaks, and estimate false contamination rates for other target fields at lower and higher redshifts. We find that this technique is highly efficient at detecting systems even down to group masses $\left(\sigma \sim 300 \mathrm{~km} \mathrm{~s}^{-1}\right)$, as well as extended structures covering significant portions of the imaging field.

The crucial component of the ORELSE Survey, and what distinguishes it from similar surveys, is the unprecedented amount of spectroscopic coverage. Utilizing the wide-field, multiobject spectrograph DEIMOS on the Keck $10 \mathrm{~m}$, we are obtaining high-quality spectra for 100-200+ confirmed members per system, allowing us to measure properties on a galaxy-to-galaxy basis. Targeting galaxies down to $i^{\prime}=24$, our system of color selection provides a spectroscopic efficiency for cluster members of up to $45 \%$.

Based on the first results from the survey, we already see significant diversity of large-scale and galaxy-scale properties at a given redshift. Although at similar redshifts, $\mathrm{Cl} 0023$ and $\mathrm{RX}$ J1821 differ in their evolutionary state, global dynamics, activity level, and galaxy population. The optically selected $\mathrm{Cl} 0023$ shows multiple high-density peaks in the red galaxy density map and a complex velocity structure, suggesting a four-way groupgroup merger. The measured velocity dispersions of the member groups range from 206 to $479 \mathrm{~km} \mathrm{~s}^{-1}$. The overall galaxy population is exceptionally active, with a high fraction of blue galaxies and [O II] emitters. Conversely, the X-ray-selected RX J1821 is a relatively isolated, centrally condensed massive cluster. Only one significant overdensity and one redshift peak are observed in this field. The measured velocity dispersion is $926 \mathrm{~km} \mathrm{~s}^{-1}$, indicative of a rich cluster. However, there are obvious signs of continuing dynamical evolution, including an elongated galaxy distribution and significant velocity substructure. The galaxy

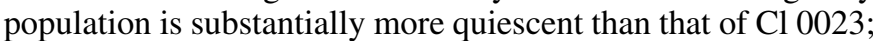
however, it still has a larger fraction of blue, star-forming galaxies than local clusters, consistent with the bluing of the cluster population with redshift. Composite spectra from both systems suggest some contribution from galaxies which have undergone a recent starburst (strong $\mathrm{H} \delta$ absorption), with the contribution in $\mathrm{Cl} 0023$ being substantial.

These two systems indicate that global cluster properties (i.e., mass) play a role in driving galaxy evolution (see also Discussion of Poggianti et al. 2006). $\mathrm{Cl} 0023$ is comprised of group-sized halos, has a small quiescent population, and subsequently contains more active galaxies. $\mathrm{Cl} 0023$ will likely be a massive cluster, with a velocity dispersion on the order of RX J1821, in $\sim 1$ Gyr, although noticeable dynamical evolution will still be taking place after $\sim 3$ Gyr, i.e., until $z \lesssim 0.3$ (Lubin et al. 1998b). If this dynamical evolution does take place, the star formation in a significant fraction of the galaxy population must be quenched during this period in order to be consistent with average SFRs and blue fractions in moderate-redshift clusters of similar mass (e.g., Balogh et al. 1997; Nakata et al. 2005). The current activity level in $\mathrm{Cl} 0023$ and this evolutionary time frame implies that the group and/or merger environment is efficient at inducing and quenching star formation. Conversely, RX J1821 is already a cluster-sized halo, with a galaxy population dominated by passive red galaxies (from both early epoch formation and later-time quenching). Although both systems will end up as massive clusters by the present day, they have clearly different evolutionary histories. We note that these results, along with preliminary findings from other ORELSE targets, suggest that $\mathrm{X}$-ray selection may be biased, at least with respect to optical surveys, toward more evolved systems at a given redshift. On the other hand, due to projection effects, optical surveys detect multigroup systems and systems embedded in large-scale structures that are not traditionally part of "cluster" surveys. As a result, the cluster-detection technique may have a strong impact on any conclusions concerning the presence and effect of large-scale structure, the relative importance of global versus local environment, and the timescales for galaxy and cluster evolution.

The scientific goals of the ORELSE Survey are to identify and examine a statistical sample of dynamically active clusters and large-scale structures during an active period in their history. Based on our completed (including spectroscopic) observations so far, such as those presented here and in Gal et al. (2008), we have found two superclusters (containing 7+ groups/clusters), a four-way group-group merger, and two largely isolated, massive X-ray-selected clusters. When the survey is complete, we will have a significant sample with which to constrain large-scale cluster dynamics, cluster formation mechanisms, and the effect of environment on galaxy evolution. Combining our ground-based databases with follow-up radio, X-ray, and/ or mid-infrared observations, the program will (1) determine the relation between global cluster properties (such as richness, mass, luminosity, and dynamical state) and the nature of the surrounding large-scale structures; (2) quantify the dependence of galaxy multiwavelength and spectral properties on position and density to determine when, where, and why star formation and nuclear activity are triggered (or truncated) in and near the cluster environment; and (3) chart the process of structure formation and its effect on galaxy evolution over this active 3 Gyr period.

We thank the anonymous referee for very constructive comments on this manuscript. This material is based upon work supported by the National Aeronautics and Space Administration under Award NNG05GC34ZG for the Long Term Space Astrophysics Program. The spectrographic data presented herein were obtained at the W. M. Keck Observatory, which is operated as a scientific partnership among the California Institute of Technology, the University of California, and the National Aeronautics and Space Administration. The Observatory was made possible by the generous financial support of the W. M. Keck Foundation. The authors recognize and acknowledge the very significant cultural role and reverence that the summit of Mauna Kea has always had within the indigenous Hawaiian community. We are most fortunate to have the opportunity to conduct observations from this mountain. The observing staff, telescope operators, and instrument scientists at Keck provided a great deal of assistance.

\section{REFERENCES}

Andreon, S., Valtchanov, I., Jones, L. R., Altieri, B., Bremer, M., Willis, J., Pierre, M., \& Quintana, H. 2005, MNRAS, 359, 1250 
Bahcall, N. A. 1988, ARA\&A, 26, 631

Balogh, M. L., Morris, S. L., Yee, H. K. C., Carlberg, R. G., \& Ellingson, E. 1997, ApJ, 488, L75

Balogh, M. L., Morris, S. L., Yee, H. K. C., Carlberg, R. G., \& Ellingson, E. 1999, ApJ, 527, 54

Balogh, M., et al. 2004, MNRAS, 348, 1355

Beers, T. C., Flynn, K., \& Gebhardt, K. 1990, AJ, 100, 32

Best, P. N. 2002, MNRAS, 336, 1293

Best, P. 2003, Ap\&SS, 285, 137

Best, P. N., van Dokkum, P. G., Franx, M., \& Rottgering, H. J. A. 2002, MNRAS, 330,17

Blakeslee, J. P., et al. 2006, ApJ, 644, 30

Blanton, E. L., Gregg, M. D., Helfand, D. J., Becker, R. H., \& White, R. L. 2003a, AJ, 125, 1635

Blanton, M. R., et al. 2003b, ApJ, 594, 186

Bower, R. G., \& Balogh, M. L. 2004, in Clusters of Galaxies: Probes of Cosmological Structure and Galaxy Evolution, ed. J. S. Mulchaey, A. Dressler, \& A. Oemler (Cambridge: Cambridge Univ. Press), 325

Branchesi, M., Gioia, I. M., Fanti, C., Fanti, R., \& Perley, R. 2006, A\&A, 446 97

Bruzual, G., \& Charlot, S. 2003, MNRAS, 344, 1000

Butcher, H., \& Oemler, A., Jr. 1984, ApJ, 285, 426

Cappelluti, N., Cappi, M., Dadina, M., Malaguti, G., Branchesi, M., D’Elia, V., \& Palumbo, G. G. C. 2005, A\&A, 430, 39

Chang, R., Gallazzi, A., Kauffmann, G., Charlot, S., Ivezić, Ž., Brinchmann, J., \& Heckman, T. M. 2006, MNRAS, 366, 717

Cohn, J. D., \& White, M. 2005, Astropart. Phys., 24, 316

Colberg, J. M., et al. 2000, MNRAS, 319, 209

Colless, M., et al. 2001, MNRAS, 328, 1039

Davis, M., Tonry, J., Huchra, J., \& Latham, D. W. 1980, ApJ, 238, L113

Davis, M., et al. 2003, Proc. SPIE, 4834, 161

De Lucia, G., et al. 2004, ApJ, 610, L77

De Lucia, G., et al. 2007, MNRAS, 374, 809

Demarco, R., et al. 2007, ApJ, 663, 164

Dolag, K., Meneghetti, M., Moscardini, L., Rasia, E., \& Bonaldi, A. 2006, MNRAS, 370, 656

Dressler, A., Oemler, A. J., Poggianti, B. M., Smail, I., Trager, S., Shectman, S. A., Couch, W. J., \& Ellis, R. S. 2004, ApJ, 617, 867

Dressler, A., \& Shectman, S. A. 1988, AJ, 95, 985

Dressler, A., Smail, I., Poggianti, B. M., Butcher, H., Couch, W. J., Ellis, R. S., \& Oemler, A. J. 1999, ApJS, 122, 51

Dressler, A., et al. 1997, ApJ, 490, 577

Eastman, J., Martini, P., Sivakoff, G., Kelson, D. D., Mulchaey, J. S., \& Tran, K.-V. 2007, ApJ, 664, L9

Ebeling, H., Barrett, E., \& Donovan, D. 2004, ApJ, 609, L49

Einasto, M., Tago, E., Jaaniste, J., Einasto, J., \& Andernach, H. 1997, A\&AS, 123,119

Eke, V. R., Navarro, J. F., \& Frenk, C. S. 1998, ApJ, 503, 569

Ellingson, E., Lin, H., Yee, H. K. C., \& Carlberg, R. G. 2001, ApJ, 547, 609

Evrard, A. E., et al. 2002, ApJ, 573, 7

Faber, S. M., et al. 2003, Proc. SPIE, 4841, 1657

Fadda, D., Biviano, A., Marleau, F. R., Storrie-Lombardi, L. J., \& Durret, F. 2008, ApJ, 672, L9

Fassbender, R., et al. 2008, A\&A, 481, 73

Finn, R. A., Zaritsky, D., \& McCarthy, D. W., Jr. 2004, ApJ, 604, 141

Fisher, D., Fabricant, D., Franx, M., \& van Dokkum, P. 1998, ApJ, 498, 195

Frenk, C. S., Evrard, A. E., White, S. D. M., \& Summers, F. J. 1996, ApJ, 472, 460

Gal, R. R., \& Lubin, L. M. 2004, ApJ, 607, L1

Gal, R. R., Lemaux, B. C., Lubin, L. M., Kocevski, D., \& Squires, G. K. 2008, ApJ, 684, 933

Gal, R. R., Lubin, L. M., \& Squires, G. K. 2005, AJ, 129, 1827

Geller, M. J., \& Huchra, J. P. 1989, Science, 246, 897

Gilbank, D. G., Yee, H. K. C., Ellingson, E., Hicks, A. K., Gladders, M. D., Barrientos, L. F., \& Keeney, B. 2008, ApJ, 677, L89

Gilmour, R., Gray, M. E., Almaini, O., Best, P., Wolf, C., Meisenheimer, K. Papovich, C., \& Bell, E. 2007, MNRAS, 380, 1467

Gioia, I. M., Henry, J. P., Mullis, C. R., Böhringer, H., Briel, U. G., Voges, W., \& Huchra, J. P. 2003, ApJS, 149, 29

Gioia, I. M., Henry, J. P., Mullis, C. R., Ebeling, H., \& Wolter, A. 1999, AJ, 117,2608

Gioia, I. M., Wolter, A., Mullis, C. R., Henry, J. P., Böhringer, H., \& Briel, U G. 2004, A\&A, 428, 867

Girardi, M., Giuricin, G., Mardirossian, F., Mezzetti, M., \& Boschin, W. 1998, ApJ, 505, 74

Gladders, M. D., \& Yee, H. K. C. 2000, AJ, 120, 2148

Gladders, M. D., \& Yee, H. K. C. 2005, ApJS, 157, 1
Gladders, M. D., Yee, H. K. C., \& Ellingson, E. 2002, AJ, 123, 1

Gómez, P. L., et al. 2003, ApJ, 584, 210

Goto, T., Yamauchi, C., Fujita, Y., Okamura, S., Sekiguchi, M., Smail, I., Bernardi, M., \& Gomez, P. L. 2003a, MNRAS, 346, 601

Goto, T., et al. 2003b, PASJ, 55, 757

Gray, M. E., Taylor, A. N., Meisenheimer, K., Dye, S., Wolf, C., \& Thommes, E. 2002, ApJ, 568, 141

Gunn, J. E., \& Gott, J. R. I. 1972, ApJ, 176, 1

Gunn, J. E., Hoessel, J. G., \& Oke, J. B. 1986, ApJ, 306, 30

Hashimoto, Y., Henry, J. P., Hasinger, G., Szokoly, G., \& Schmidt, M. 2005, A\&A, 439, 29

Henry, J. P., Mullis, C. R., Voges, W., Böhringer, H., Briel, U. G., Gioia, I. M., \& Huchra, J. P. 2006, ApJS, 162, 304

Holden, B. P., Stanford, S. A., Squires, G. K., Rosati, P., Tozzi, P., Eisenhardt, P., \& Spinrad, H. 2002, AJ, 124, 33

Homeier, N. L., et al. 2006, ApJ, 647, 256

Howell, S. B., \& Tonry, J. L. 2003, BAAS, 35, 1235

Jannuzi, B. T., et al. 2004, BAAS, 36, 1478

Jeltema, T. E., Canizares, C. R., Bautz, M. W., \& Buote, D. A. 2005, ApJ, 624 606

Kaiser, N., Wilson, G., Luppino, G., \& Dahle, H. 1999, arXiv:astro-ph/9907229

Kartaltepe, J. S., Ebeling, H., Ma, C. J., \& Donovon, D. 2008, MNRAS, 389, 1240

Kocevski, D. D., Lubin, L. M., Gal, R., Lemaux, B. C., Fassnacht, C. D., \& Squires, G. K. 2009a, ApJ, 690, 295

Kocevski, D. D., Lubin, L. M., Lemaux, B. C., Gal, R., Fassnacht, C. D., Lin, R., \& Squires, G. K. 2009b, ApJ, in press

Kocevski, D. D., Lubin, L. M., Lemaux, B. C., Gal, R., Fassnacht, C. D., Lin, R., \& Squires, G. K. 2009c, ApJ, submitted

Kodama, T., \& Arimoto, N. 1997, A\&A, 320, 41

Kodama, T., Smail, I., Nakata, F., Okamura, S., \& Bower, R. G. 2001, ApJ, 562 , L9

Kodama, T., Smail, I., Nakata, F., Okamura, S., \& Bower, R. G. 2003, ApJ, 591, L169

Kodama, T., et al. 2004, MNRAS, 350, 1005

Kodama, T., et al. 2005, PASJ, 57, 309

Koekemoer, A. M., et al. 2007, ApJS, 172, 196

Koester, B. P., et al. 2007, ApJ, 660, 221

Koyama, Y., Kodama, T., Tanaka, M., Shimasaku, K., \& Okamura, S. 2007, MNRAS, 382, 1719

Larson, R. B., Tinsley, B. M., \& Caldwell, C. N. 1980, ApJ, 237, 692

Lewis, I., et al. 2002, MNRAS, 334, 673

Lopes, P. A. A., de Carvalho, R. R., Capelato, H. V., Gal, R. R., Djorgovski, S G., Brunner, R. J., Odewahn, S. C., \& Mahabal, A. A. 2006, ApJ, 648, 209

Lubin, L. M., Brunner, R., Metzger, M. R., Postman, M., \& Oke, J. B. 2000, ApJ, 531, L5

Lubin, L. M., Mulchaey, J. S., \& Postman, M. 2004, ApJ, 601, L9

Lubin, L. M., Oke, J. B., \& Postman, M. 2002, AJ, 124, 1905

Lubin, L. M., Postman, M., Oke, J. B., Ratnatunga, K. U., Gunn, J. E., Hoessel, J. G., \& Schneider, D. P. 1998a, AJ, 116, 584

Lubin, L. M., Postman, M., \& Oke, J. B. 1998b, AJ, 116, 643

Marcillac, D., Rigby, J. R., Rieke, G. H., \& Kelly, D. M. 2007, ApJ, 654, 825

Marcillac, D., et al. 2008, ApJ, 675, 1156

Margoniner, V. E., \& Wittman, D. M. 2008, ApJ, 679, 31

Maughan, B. J., Jones, L. R., Ebeling, H., \& Scharf, C. 2006, MNRAS, 365, 509

Mei, S., et al. 2006a, ApJ, 639, 81

Mei, S., et al. 2006b, ApJ, 644, 759

Mihos, C. 1999, Ap\&SS, 266, 195

Mihos, J. C. 1995, ApJ, 438, L75

Mihos, J. C. 2004, in Clusters of Galaxies: Probes of Cosmological Structure and Galaxy Evolution, ed. J. S. Mulchaey, A. Dressler, \& A. Oemler (Cambridge: Cambridge Univ. Press), 277

Miller, N. A., \& Owen, F. N. 2003, AJ, 125, 2427

Moore, B., Lake, G., \& Katz, N. 1998, ApJ, 495, 139

Mullis, C. R., et al. 2003, ApJ, 594, 154

Nakata, F., et al. 2005, MNRAS, 357, 1357

Oke, J. B., Postman, M., \& Lubin, L. M. 1998, AJ, 116, 549

Oke, J. B., et al. 1995, PASP, 107, 375

Owen, F. N., Ledlow, M. J., Keel, W. C., \& Morrison, G. E. 1999, AJ, 118, 633

Patel, S. G., Kelson, D. D., Holden, B. P., Illingworth, G. D., Franx, M., van der Wel, A., \& Ford, H. 2009, ApJ, 694, 1349

Pierre, M., et al. 2006, MNRAS, 372, 591

Poggianti, B. M., et al. 2006, ApJ, 642, 188

Porter, S. C., Raychaudhury, S., Pimbblet, K. A., \& Drinkwater, M. J. 2008, MNRAS, 388, 1152

Postman, M., Geller, M. J., \& Huchra, J. P. 1988, AJ, 95, 267 
Postman, M., Lubin, L. M., Gunn, J. E., Oke, J. B., Hoessel, J. G., Schneider D. P., \& Christensen, J. A. 1996, AJ, 111, 615

Postman, M., Lubin, L. M., \& Oke, J. B. 1998, AJ, 116, 560

Quintana, H., Ramirez, A., Melnick, J., Raychaudhury, S., \& Slezak, E. 1995, AJ, 110, 463

Rines, K., Geller, M. J., Diaferio, A., Mahdavi, A., Mohr, J. J., \& Wegner, G. 2002, AJ, 124, 1266

Rosati, P., della Ceca, R., Norman, C., \& Giacconi, R. 1998, ApJ, 492, L2

Rosati, P., Stanford, S. A., Eisenhardt, P. R., Elston, R., Spinrad, H., Stern, D., \& Dey, A. 1999, AJ, 118, 76

Scoville, N., et al. 2007, ApJS, 172, 1

Simcoe, R. A., Metzger, M. R., Small, T. A., \& Araya, G. 2000, BAAS, 32, 758

Smail, I., Edge, A. C., Ellis, R. S., \& Blandford, R. D. 1998, MNRAS, 293, 124

Small, T. A., Ma, C.-P., Sargent, W. L. W., \& Hamilton, D. 1998, ApJ, 492, 45

Stanford, S. A., Eisenhardt, P. R., \& Dickinson, M. 1998, ApJ, 492, 461

Stanford, S. A., Holden, B., Rosati, P., Eisenhardt, P. R., Stern, D., Squires, G., \& Spinrad, H. 2002, AJ, 123, 619

Swinbank, A. M., et al. 2007, MNRAS, 379, 1343

Tanaka, M., Goto, T., Okamura, S., Shimasaku, K., \& Brinkmann, J. 2004, AJ, 128,2677

Tanaka, M., Hoshi, T., Kodama, T., \& Kashikawa, N. 2007a, MNRAS, 379, 1546
Tanaka, M., Kodama, T., Arimoto, N., Okamura, S., Umetsu, K., Shimasaku, K., Tanaka, I., \& Yamada, T. 2005, MNRAS, 362, 268

Tanaka, M., Kodama, T., Arimoto, N., \& Tanaka, I. 2006, MNRAS, 365, 1392

Tanaka, M., Kodama, T., Kajisawa, M., Bower, R., Demarco, R., Finoguenov, A., Lidman, C., \& Rosati, P. 2007b, MNRAS, 377, 1206

Tody, D. 1986, Proc. SPIE, 627, 733

Tran, K.-V. H., Franx, M., Illingworth, G., Kelson, D. D., \& van Dokkum, P. 2003, ApJ, 599, 865

Treu, T., Ellis, R. S., Kneib, J.-P., Dressler, A., Smail, I., Czoske, O., Oemler, A., \& Natarajan, P. 2003, ApJ, 591, 53

Valtchanov, I., et al. 2004, A\&A, 423, 75

van Dokkum, P. G., \& Franx, M. 2001, ApJ, 553, 90

van Dokkum, P. G., Franx, M., Fabricant, D., Illingworth, G. D., \& Kelson, D. D. 2000, ApJ, 541, 95

van Dokkum, P. G., Stanford, S. A., Holden, B. P., Eisenhardt, P. R., Dickinson M., \& Elston, R. 2001, ApJ, 552, L101

Vikhlinin, A., McNamara, B. R., Forman, W., Jones, C., Quintana, H., \& Hornstrup, A. 1998, ApJ, 498, L21

Yan, R., Newman, J. A., Faber, S. M., Konidaris, N., Koo, D., \& Davis, M 2006, ApJ, 648, 281

Zabludoff, A. I., \& Mulchaey, J. S. 1998, ApJ, 496, 39 